\title{
Ultrashort echo time (UTE) magnetic resonance imaging of myelin: technical developments and challenges
}

\author{
Ya-Jun Ma ${ }^{1}$, Hyungseok Jang ${ }^{1}$, Eric Y. Chang ${ }^{1,2}$, Annie Hiniker ${ }^{3}$, Brian P. Head ${ }^{4}$, Roland R. Lee ${ }^{1}$, \\ Jody Corey-Bloom ${ }^{5}$, Graeme M. Bydder ${ }^{1}$, Jiang $\mathrm{Du}^{1}$ \\ ${ }^{1}$ Department of Radiology, University of California San Diego, San Diego, CA, USA; ${ }^{2}$ Radiology Service, VA San Diego Healthcare System, San \\ Diego, CA, USA; ${ }^{3}$ Department of Pathology, ${ }^{4}$ Department of Anesthesiology, ${ }^{5}$ Department of Neurosciences, University of California San Diego, \\ San Diego, CA, USA
}

Correspondence to: Jiang Du, PhD. Department of Radiology, University of California San Diego, La Jolla, CA 92037, USA. Email: jiangdu@ucsd.edu.

Provenance and Peer Review: This article was commissioned by the editorial office, Quantitative Imaging in Medicine and Surgery. The article did not undergo external peer review.

Submitted Apr 10, 2020. Accepted for publication Apr 23, 2020.

doi: 10.21037/qims-20-541

View this article at: http://dx.doi.org/10.21037/qims-20-541

\section{Introduction}

Myelin is a concentrically laminated membranous structure consisting of alternating protein and lipid layers, and contains approximately $20 \%$ protein and $80 \%$ lipid (1). It is formed by two different types of support cells, oligodendrocytes in the central nervous system (CNS) and Schwann cells in the peripheral nervous system (PNS). Myelin is a major component of the CNS and PNS, and accounts for about $14 \%$ of the wet mass and $50 \%$ of the dry mass of the white matter of the brain (1). It is present in the form of a sheath surrounding the axons of some neurons, and insulates axons from electrically charged ions and molecules. It helps increase nerve conduction velocity. Loss of myelin is the hallmark of numerous inflammatory and neurodegenerative disorders, including multiple sclerosis (MS) and other demyelinating diseases such as optic neuritis, neuromyelitis optica, transverse myelitis, and acute disseminated encephalomyelitis (1).

Magnetic resonance imaging (MRI) is the current gold standard imaging modality for diagnosis of disease of the brain (2-10). Conventional clinical MRI sequences are very sensitive to the presence of white matter disease including MS. Clinical $\mathrm{T}_{1^{-}}$and $\mathrm{T}_{2}$-weighted fast spin echo (FSE) imaging (2), gadolinium enhancement (3), diffusion tensor imaging (DTI) (4), and Magnetization Transfer (MT) (5), all show high sensitivity for abnormalities in patients with
MS. However, conventional clinical MRI only correlates modestly with disability assessed by the expanded disability status scale (EDSS) (6), also $\mathrm{T}_{2}$-hyperintense lesion load in MS is poorly correlated with disability $(\mathrm{r}=0.2-0.5)$ in crosssectional studies (7-11). Contrast-enhanced lesions are only moderately correlated with disability in the first six months, and are not predictive of changes in the EDSS in the subsequent 12 or 24 months (12). A recent large scale multicenter study reported a poor correlation between EDSS and normalized brain volume $(\mathrm{r}=-0.18)$, cross section area $(\mathrm{r}=-0.26)$, MT ratio (MTR) of whole brain tissue $(\mathrm{r}=-0.16)$ and MTR of gray matter (GM) (r=-0.17), and no significant correlation between other MR metrics and patients EDSS scores (13).

It is commonly accepted that conventional clinical MRI sequences lack specificity for evaluation of the heterogeneous pathologic substrates of MS as well as the ability to provide accurate estimates of damage in areas of the brain apart from focal lesions (6). Most conventional clinical MRI sequences cannot distinguish the different cardinal pathological substrates of MS, namely demyelination, remyelination, inflammation, edema, axonal loss and gliosis (14-16). The inability of conventional MRI sequences to distinguish demyelination and remyelination may be a major factor accounting for the poor correlation between regular MRI metrics and disability. 
Direct assessment of the integrity of myelin in the CNS and PNS may be important for the diagnosis and assessment of prognosis in many demyelinating diseases such as MS. However, the protons in myelin have extremely short $T_{2} \mathrm{~s}$ (less than $1 \mathrm{~ms}$ ) (17), and cannot be directly imaged with conventional clinical MRI sequences which have TEs of several milliseconds or longer. As a result, conventional clinical sequences only provide an indirect assessment of myelin. Myelin specific information, such as its $T_{1}$ and $\mathrm{T}_{2}{ }^{*}$ as well as its proton density are not widely known. Ultrashort echo time (UTE) sequences with minimum nominal TEs of 8-100 $\mu$ s, which are 100 times shorter than the TEs of conventional clinical sequences, make it possible to directly detect signal from myelin using whole body clinical MRI scanners (18-28). Herein we review recent technical developments in UTE imaging of myelin ex vivo and in vivo. Challenges associated with morphological and quantitative myelin imaging are also discussed.

\section{Contrast mechanisms}

The basic 2D UTE sequence employs a half pulse or short rectangular pulse for signal excitation, followed by radial mapping of k-space from the center out. Two excitations are performed with the gradient polarity reversed on the second occasion. The data from these excitations are added to produce a single radial line of $\mathrm{k}$-space. Raw data is reconstructed after re-gridding using inverse Fourier transformation. As myelin represents a small fraction of the total signal from the brain, the dominant signal is from long $\mathrm{T}_{2}$ white matter $\left(\mathrm{WM}_{\mathrm{L}}\right)$ and long $\mathrm{T}_{2}$ gray matter $\left(\mathrm{GM}_{\mathrm{L}}\right)$. It is essential to suppress those signals in order to generate high contrast specific images of myelin.

Adiabatic inversion recovery prepared dual echo UTE (IR-UTE) sequences have been used for selective myelin imaging. With this approach, a relatively long (e.g., duration $=8.64 \mathrm{~ms}$ ) adiabatic fast passage (SilverHoult) inversion pulse is used to invert the longitudinal magnetization of $W M_{L}$ and $\mathrm{GM}_{\mathrm{L}}$ (18). The longitudinal magnetization of myelin with its extremely short $T_{2}{ }^{*}\left(M_{S}\right)$ is saturated due to fast transverse relaxation during the long adiabatic inversion process (29). UTE data acquisition starts at the inversion time (TI) necessary for the inverted longitudinal magnetization of $\mathrm{WM}_{\mathrm{L}}$ to reach its null point, leaving signals from myelin and some long $\mathrm{T}_{2}$ tissues (e.g., $\mathrm{GM}_{\mathrm{L}}$ ) to be detected by a dual echo initial UTE, and later gradient echo data acquisition. The second echo acquires signals from the non-nulled long $T_{2}$ tissues such as $G_{L}$.
The second echo image shows zero or near zero signal from myelin. Subtraction of the second echo image from the first one provides selective imaging of myelin in white matter. Figure 1 describes the contrast mechanism. Note that on the subtracted image myelin in white matter has a positive signal while ultrashort $T_{2}$ components in gray matter have a negative signal.

The same contrast mechanisms can be directly used in 3D UTE imaging. More recently, a 3D UTE Cones sequence together with adiabatic inversion recovery preparation (3D IR-UTE-Cones) has been implemented for fast volumetric imaging of short $T_{2}$ species such as cortical bone (30). In this sequence, a short rectangular pulse is used for excitation followed by a cones trajectory with a minimal nominal TE of $32 \mu$ s (a minimal TE of 8 us can be achieved with the use of a fast transmit/receive switch). The $3 \mathrm{D} \mathrm{k}$-space is divided into multiple cones, with twisted radial trajectories along each cone. The $3 \mathrm{D}$ Cones sequences minimize the problem with eddy currents associated with 2D UTE sequences. Furthermore, the 3D Cones sequence allows use of anisotropic field of view (FOV) and spatial resolution, which can greatly reduce the total scan time (30). Volumetric imaging of ultrashort T2 species can be achieved in a few minutes with the $3 \mathrm{D}$ IR-UTECones sequence (31).

\section{Validation with myelin phantom studies}

The capability of UTE MRI for direct imaging of myelin has been demonstrated in a series of myelin phantom studies. Figure 2 shows UTE imaging of a $90 \%$ purified lyophilized bovine myelin extract powder (type 1 bovine brain lipid extract, Sigma-Aldrich B1502, St. Louis, MO, USA) and a mixture of it with $99.9 \% \mathrm{D}_{2} \mathrm{O}$ (Sigma-Aldrich) in the form of a paste. The $\mathrm{T}_{2}{ }^{*}$ of myelin powder was slightly longer than that of the myelin- $\mathrm{D}_{2} \mathrm{O}$ paste probably because of the greater susceptibility of myelin in powder form.

The myelin lipid powder was resuspended in $\mathrm{D}_{2} \mathrm{O}$ at concentrations of $6 \%, 9 \%, 12 \%, 18 \%$, and $24 \% \mathrm{w} / \mathrm{v}$ to approximate the physiological range of myelin concentrations in white matter. The samples were loaded into small syringes along with a $\mathrm{D}_{2} \mathrm{O}$-only control $(0 \%$ myelin) prior to imaging. After normalization relative to a homogeneous myelin free $\mathrm{D}_{2} \mathrm{O}$ phantom, the normalized UTE signal intensity showed a highly linear increase with myelin concentration ( $\mathrm{R}^{2}$ of $99 \%$ ) as seen in Figure 3 (31). This high linearity is very similar to the result obtained by Siefert et al. using a $9.4 \mathrm{~T}$ spectrometer, with ZTE 

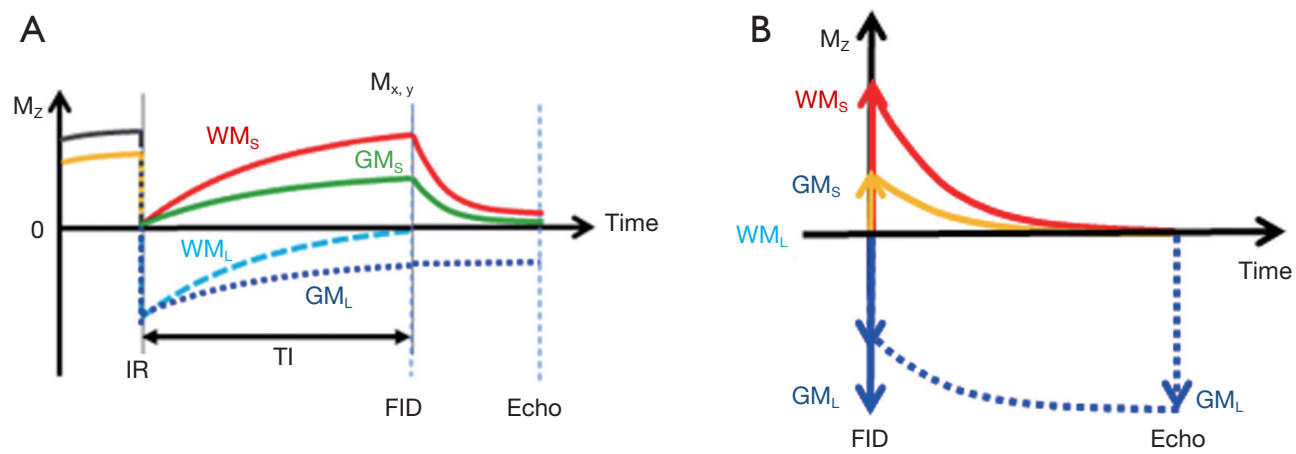

Figure 1 Contrast mechanism of myelin imaging using IR-UTE sequences. (A) Illustration of the contrast mechanisms in imaging ultrashort $T_{2}$ components (such as myelin) in white matter $\left(W_{\mathrm{S}}\right)$ using a IR-UTE sequence with an inversion time (TI) set for nulling of signals from the long $T_{2}$ components in white matter $\left(W M_{L}\right)$. The long $T_{2}$ components in gray matter $\left(G M_{L}\right)$ have negative longitudinal magnetization at the time of the initial free induction decay (FID) data acquisition because $\mathrm{GM}_{\mathrm{L}}$ has a longer $\mathrm{T} 1$ than $W M_{\mathrm{L}}$. Myelin has an extremely short $T_{2}\left(T_{2}<1 \mathrm{~ms}\right)$, which is far shorter than the duration of the adiabatic inversion pulse (duration =8.64 ms), and so its longitudinal magnetization is saturated by the long adiabatic IR pulse. It subsequently recovers relatively quickly because its T1 is shorter than those of $W M_{L}$ and $G_{L}$. As a result at the null point, the white matter signal only comes from $W_{S}$. However, gray matter is more complicated: there is a cancellation between positive longitudinal magnetization from ultrashort $\mathrm{T}_{2}$ components (e.g., myelin) in gray matter (GMS) and negative longitudinal magnetization from $\mathrm{GM}_{\mathrm{L}}$ producing a net reduction in transverse magnetization at the FID after the excitation pulse. At the $2^{\text {nd }}$ echo (e.g., TE $\sim 2 \mathrm{~ms}$ ), the myelin signal in gray matter decays to zero or near zero, while the signal from $\mathrm{GM}_{\mathrm{L}}$ decays much less due to its longer $\mathrm{T}_{2}{ }^{*}$, so the net signal of the transverse magnetization is greater at the $2^{\text {nd }}$ echo than at the initial one. As a result, GM has a higher signal at the $2^{\text {nd }}$ echo than at the $1^{\text {st }}$ echo or FID (B). Subtraction of the $2^{\text {nd }}$ echo from the $1^{\text {st }}$ one (both magnitude reconstructed) leads to a positive signal for myelin in white matter, but a negative signal for ultrashort $\mathrm{T}_{2}$ components (including myelin) in gray matter, which creates very high myelin contrast.
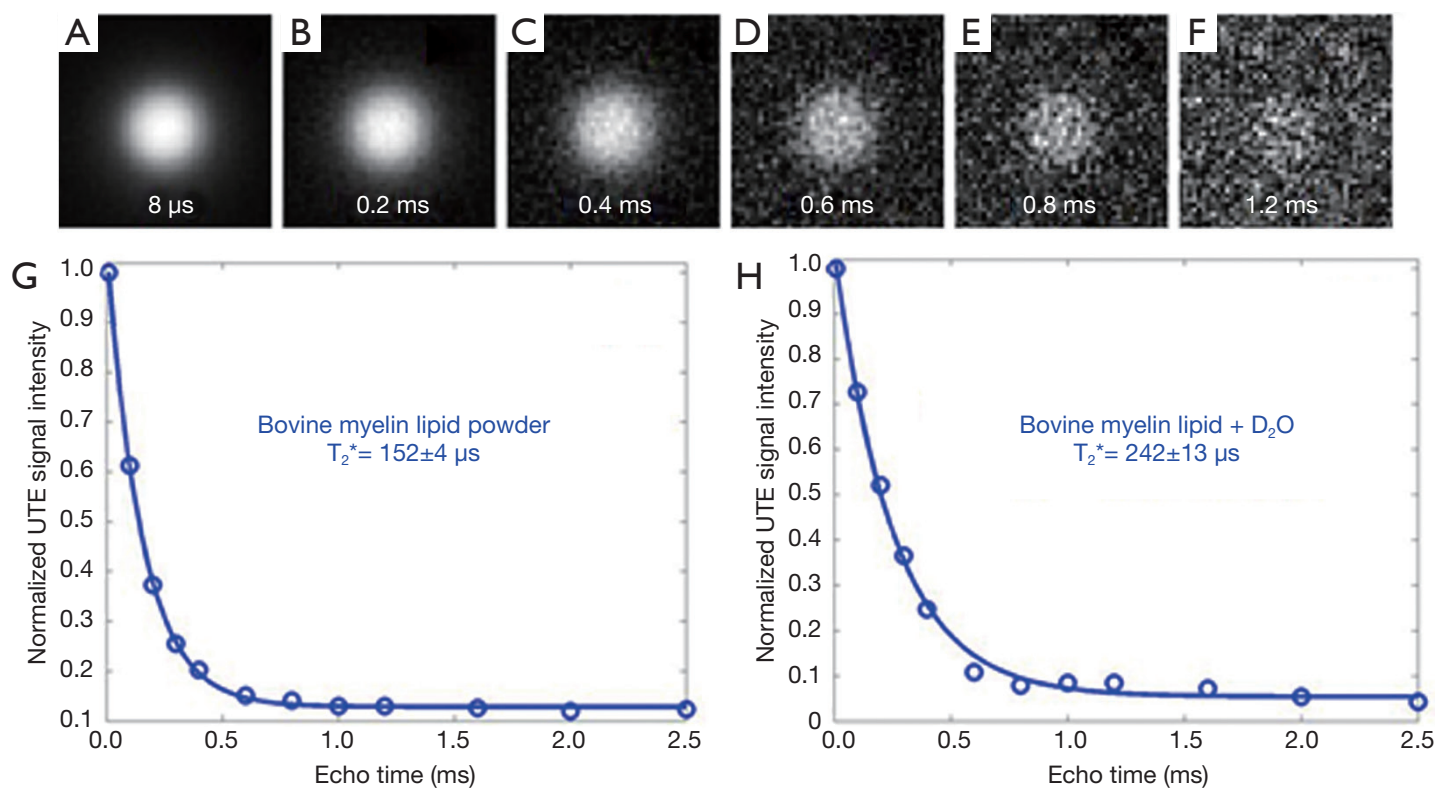

Figure 2 UTE imaging of bovine myelin lipid powder with TEs of $8 \mu$ s (A), 0.2 (B), 0.4 (C), 0.6 (D), 0.8 (E) and 1.2 ms (F). The powder shows excellent single-component signal decay with an ultrashort $\mathrm{T}_{2}{ }^{*}$ of $152 \pm 4 \mu \mathrm{s}(\mathrm{G})$. Similar behavior with a $\mathrm{T}_{2}{ }^{*}$ of $242 \pm 13 \mu \mathrm{s}$ was demonstrated for the bovine myelin lipid- $\mathrm{D}_{2} \mathrm{O}$ paste $(\mathrm{H})$. Adapted from (23), with permission. 

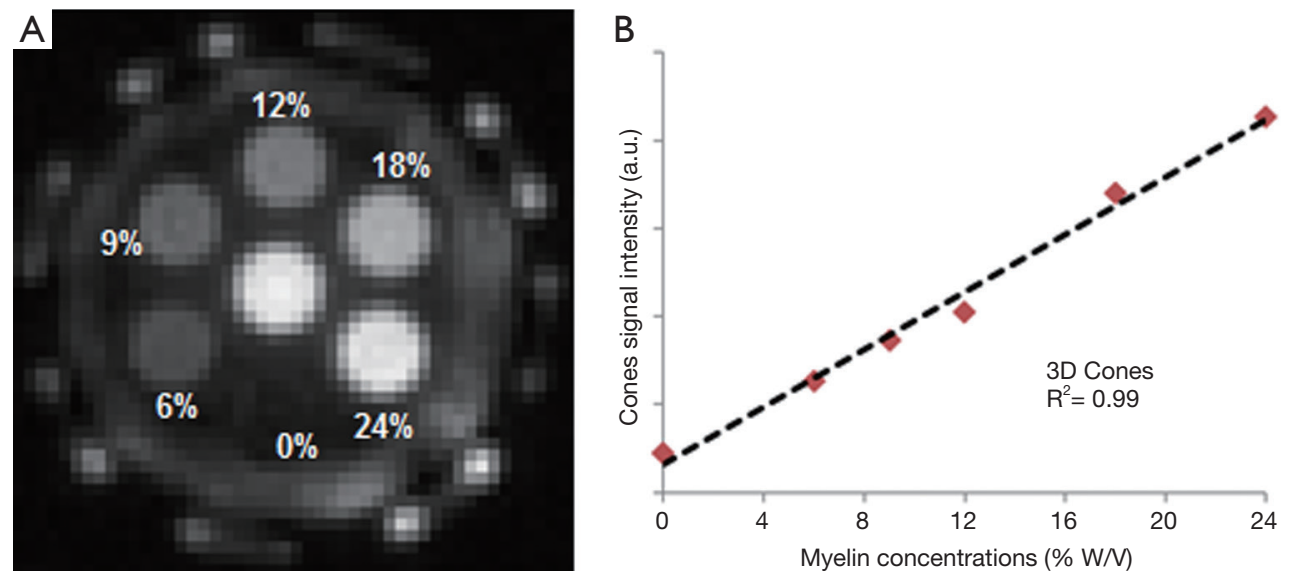

Figure 3 Correlation between UTE signals and myelin concentrations. (A) 3D-UTE-Cones imaging of myelin phantoms with myelin concentrations ranging from 0-24\%. (B) Linear regression analysis shows a high correlation between myelin concentration and 3D-UTECones signals (R2 =0.99) (B), demonstrating that the 3D-UTE-Cones sequence can directly detect and quantify myelin protons. Adapted from (31), with permission.

signal detection. In their studies signal amplitudes were linearly correlated with myelin concentration with an $\mathrm{R}^{2}$ of 0.98-0.99 (32), demonstrating the detectability of myelin and the potential for in vivo myelin imaging on clinical MR scanners. In our study, a single-component relaxation curve was found at each myelin concentration (6-24\%), with fitted $\mathrm{T}_{2}{ }^{*}$ values of $\sim 0.3 \mathrm{~ms}$.

Myelin powder in $\mathrm{D}_{2} \mathrm{O}$ or $\mathrm{H}_{2} \mathrm{O}$ may not have myelin's typical physiological bilayer structure. Myelin isolated from porcine white matter by mechanical homogenization was therefore studied to assess myelin with its membrane architecture preserved. Myelin vesicles obtained in this way were purified using discontinuous sucrose gradient ultracentrifugation (33). After thoroughly washing out residual sucrose, the myelin vesicles were resuspended twice in deuterated tris-Cl buffer to remove residual $\mathrm{H}_{2} \mathrm{O}$. UTE images showed high signal from the intact myelin vesicles. These had a short $T_{1}$ of $367 \pm 4 \mathrm{~ms}$ and an ultrashort $\mathrm{T}_{2}{ }^{*}$ of $225 \pm 7 \mu$ s (Figure 4). This result showed that intact myelin vesicles can be directly imaged and quantified with $3 \mathrm{D}$ UTE sequences using a clinical $3 \mathrm{~T}$ scanner.

\section{Validation with brain specimen studies}

MS brain specimens have been imaged with both IRUTE and conventional clinical MRI sequences, and then subjected to histopathologic analysis. Figure 5 shows representative clinical and IR-UTE images of the brain from a 28-year-old female donor with confirmed MS (23).
Areas of normal appearing white matter (NAWM) appear high signal on the IR-UTE subtracted images, and areas of abnormality appear low signal. Some lesions seen on the IR-UTE images correspond to $T_{2}$ hyperintense areas on conventional $\mathrm{T}_{2}$-weighted images and hypointense areas on $\mathrm{T}_{2}$-FLAIR images, but the abnormalities seen on the IRUTE images extended into the adjacent areas of NAWM seen with the conventional images. The IR-UTE sequence showed areas of abnormality in locations that appeared normal with conventional $\mathrm{T}_{2}$-weighted and $\mathrm{T}_{2}$-FLAIR images.

Comparison of typical clinical sequences and an IRUTE-Cones sequence in the ex vivo MS brain study is shown in Figure 6 (31). The bright signals in IR-UTECones images have $T_{2}{ }^{*} \mathrm{~s}$ around $0.20 \pm 0.04 \mathrm{~ms}$, which is close to the $\mathrm{T}_{2}{ }^{*}$ of myelin- $\mathrm{D}_{2} \mathrm{O}$ phantom shown in Figures 2,4, consistent with myelin protons as the signal source. MS lesions identified in the clinical images show myelin signal loss in corresponding regions in the IR-UTE-Cones images. Representative lesions were subsequently confirmed as demyelinated on histology (Figure 6B).

Wilhelm et al. implemented a similar technique on a Bruker 9.4 $\mathrm{T}$ scanner (20). They investigated IR-UTE imaging and NMR spectroscopy of fresh rat spinal cord before and after $\mathrm{D}_{2} \mathrm{O}$ exchange. Figure 7 shows some of their results at 9.4T. A major problem with their study is that IR-UTE images were acquired at a TI empirically selected to null long $\mathrm{T}_{2}$ white matter signal. At $\mathrm{TE}_{2}$ $=1,200 \mu$ s (Figure 7B), the WM signal should be zero, or 

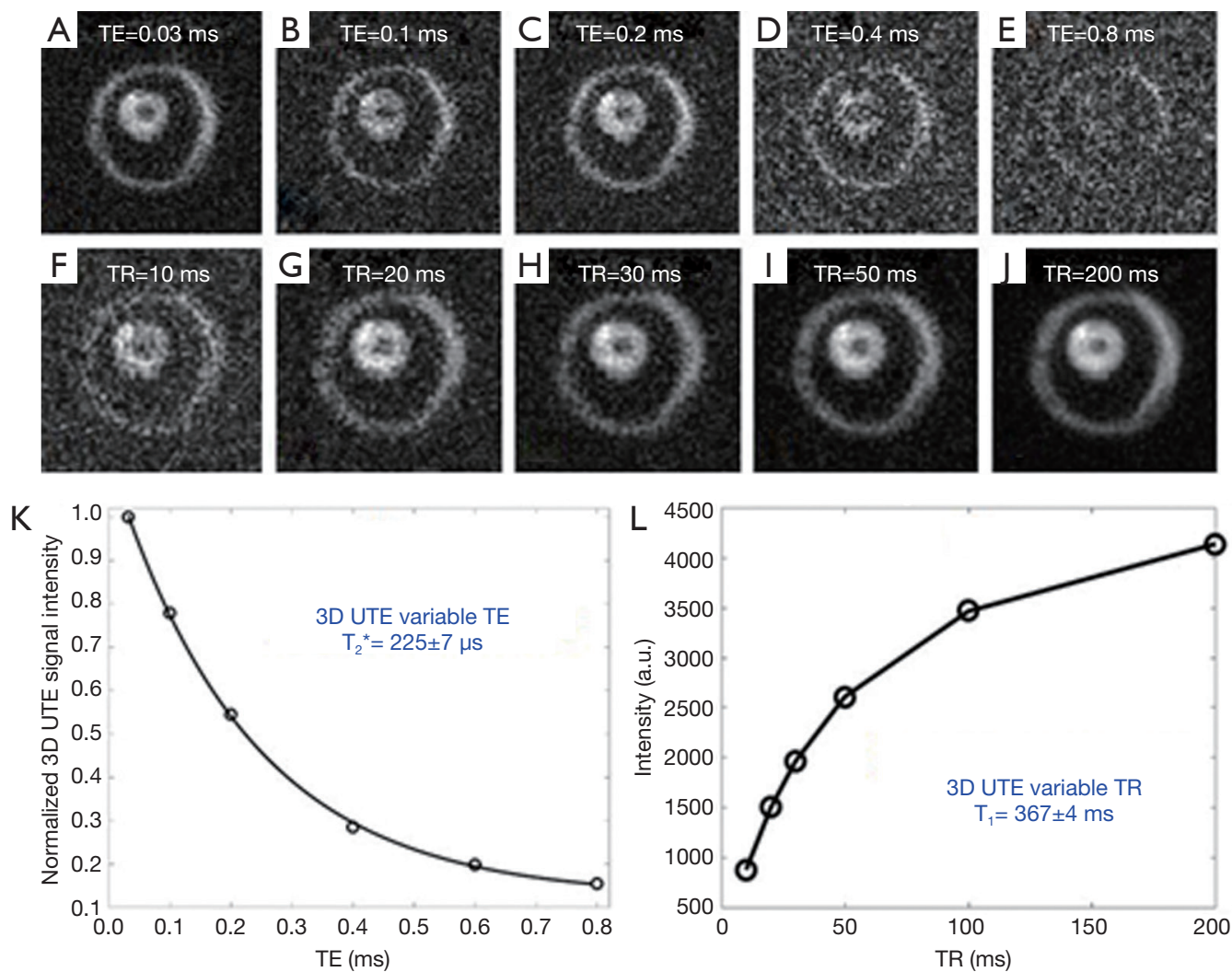

Figure 4 Quantitative $T_{2}{ }^{*}$ and $T_{1}$ measurement of the myelin vesicle phantom.(A) 3D UTE imaging of intact myelin vesicles with TEs of 0.03 (A), 0.1 (B), 0.2 (C), 0.4 (D), and $0.8 \mathrm{~ms}(\mathrm{E})$, and TRs of 10 (F), 20 (G), 30 (H), 50 (I) and $200 \mathrm{~ms}(\mathrm{~J})$. The corresponding $\mathrm{T}_{2}{ }^{*}$ and $\mathrm{T}_{1}$ fitting show a $\mathrm{T}_{2}{ }^{*}$ of $225 \pm 7 \mu \mathrm{s}(\mathrm{K})$ and a $\mathrm{T}_{1}$ of $367 \pm 4 \mathrm{~ms}(\mathrm{~L})$. From (34), with permission.
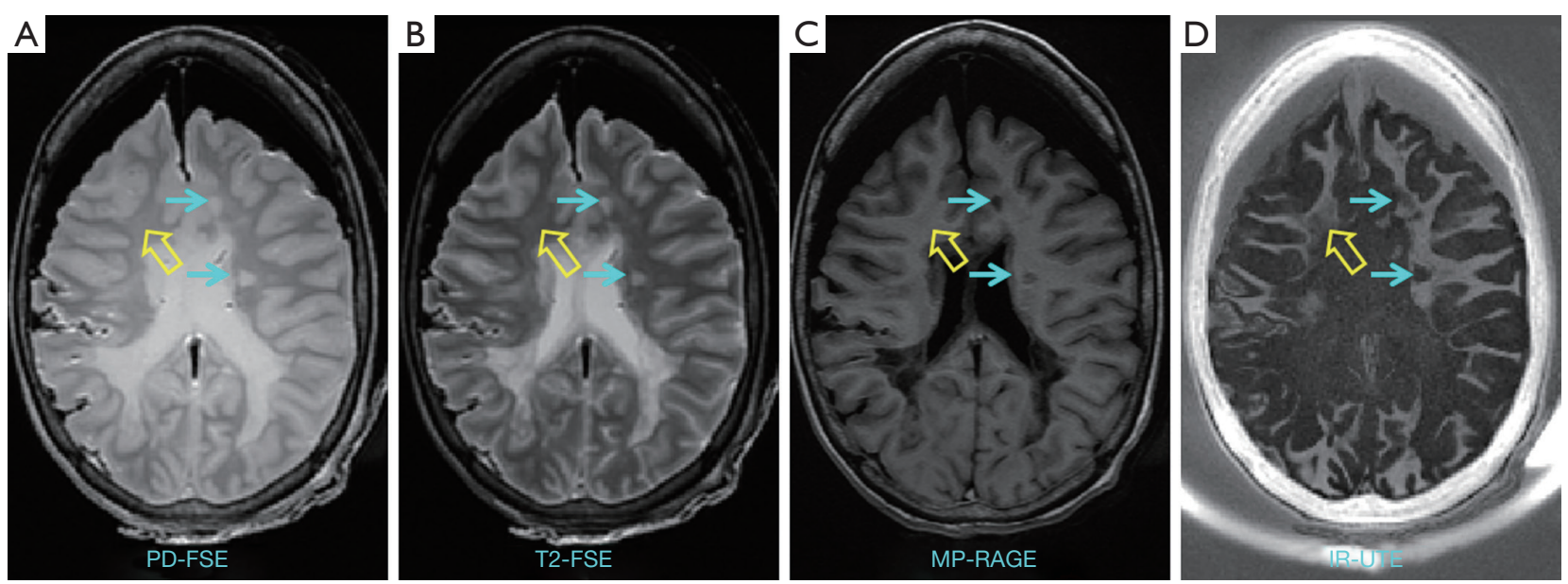

Figure 5 Clinical PD-FSE (A), $\mathrm{T}_{2}$-FSE (B) and MP-RAGE (C) imaging as well as IR-UTE (D) imaging of a brain specimen from a 28 year old female donor with confirmed MS. MS lesions are hyperintense (thin arrows, A, B) on the PD-FSE and T $-F S E$ images, and hypointense (thin arrows, C) on the MP-RAGE image, and show signal loss on the IR-UTE image (thin arrows, D). Complete myelin loss is obvious in regions indicated by the thin arrows. Partial loss of signal is seen in the IR-UTE image (thick arrow, D) where the PD-FSE, T $-F S E$ and MP-RAGE images appear normal (thick arrows, A-C). Adapted from (23), with permission. 
A
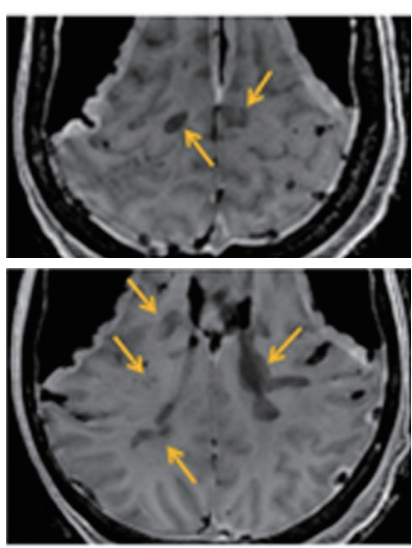

$\mathrm{T}_{2}$-FSE
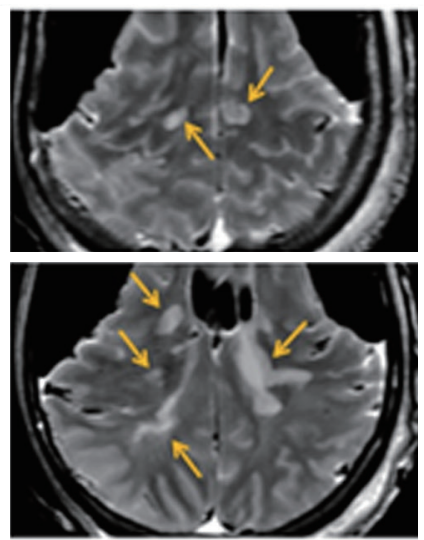

IR-UTE-Cones
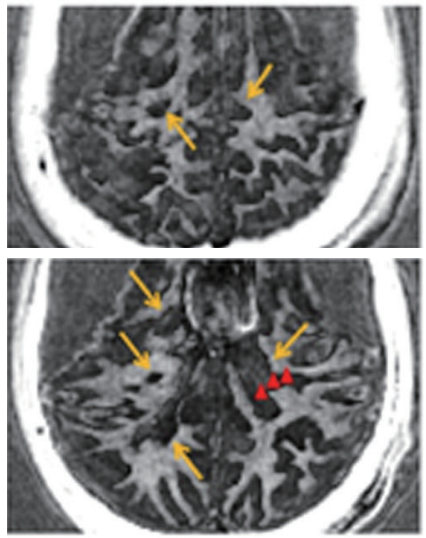

B
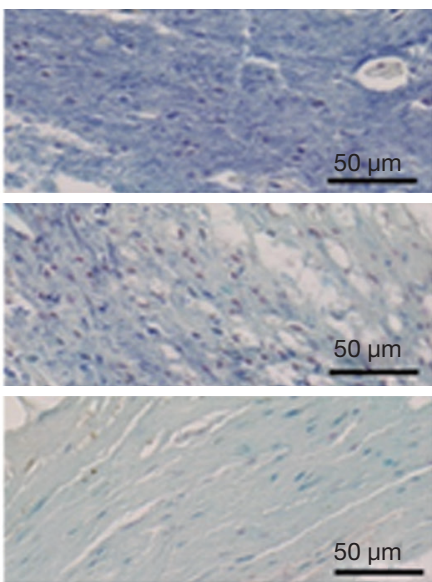

Figure 6 Representative ex vivo MS brain images (45-year-old male donor) with a high disease burden using clinical $\mathrm{T}_{1}$-weighted MP-RAGE, $\mathrm{T}_{2}$-weighted FSE, and 3D IR-UTE-Cones (A). Arrows point to MS lesions on all three images. The 3D IR-UTE-Cones sequence shows signal loss in MS lesions. Representative histology of sample MS lesions using Luxol Fast Blue as a myelin stain, counterstained with neutral red is shown in (B). Regions of NAWM (top), lesion edge (middle), and central lesion (bottom) demonstrate specific loss of myelin staining in the MS lesions. The three red triangular arrowheads in (A) show the locations of the histological samples. Adapted from (31), with permission.

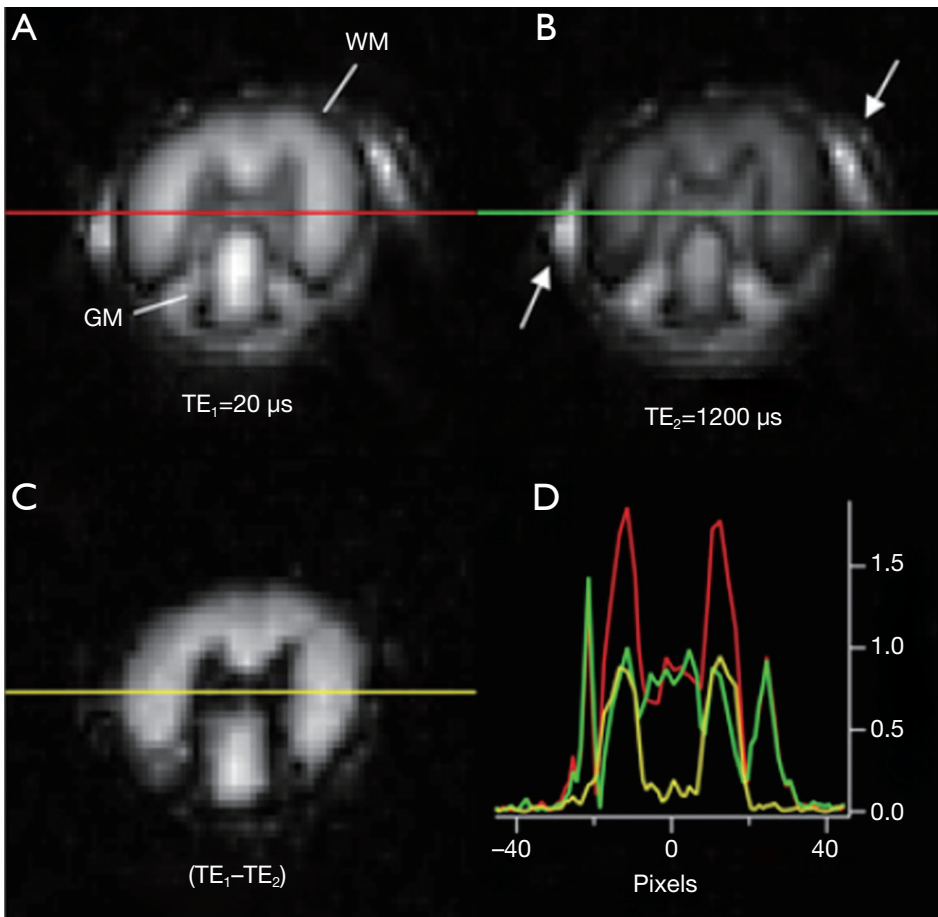

Figure 7 3D de-IR-UTE images from rat thoracic spinal cord (SC) averaged over five central slices. Magnitude images obtained for (A) TE $=20 \mu \mathrm{s},(\mathrm{B}) \mathrm{TE}=1,200 \mu \mathrm{s}$, and (C) complex difference (A - B) (maximum-intensity range decreased by a factor of two to highlight myelin signal). (D) Intensity profiles across the three images (delineated as red, green, and yellow lines in A, B, and C, respectively) to show relative WM, GM, and background intensity. The most intense signal, present in the short- and long-echo profiles, originates from residual surface water. WM and GM are indicated in A, and arrows highlight residual surface water in B. The dark boundary observed at the GM/WM and $\mathrm{WM} /$ surface water interfaces in both echo images stems from partial voluming of adjacent regions with different T1s resulting in destructive interference. From (20), with permission. 

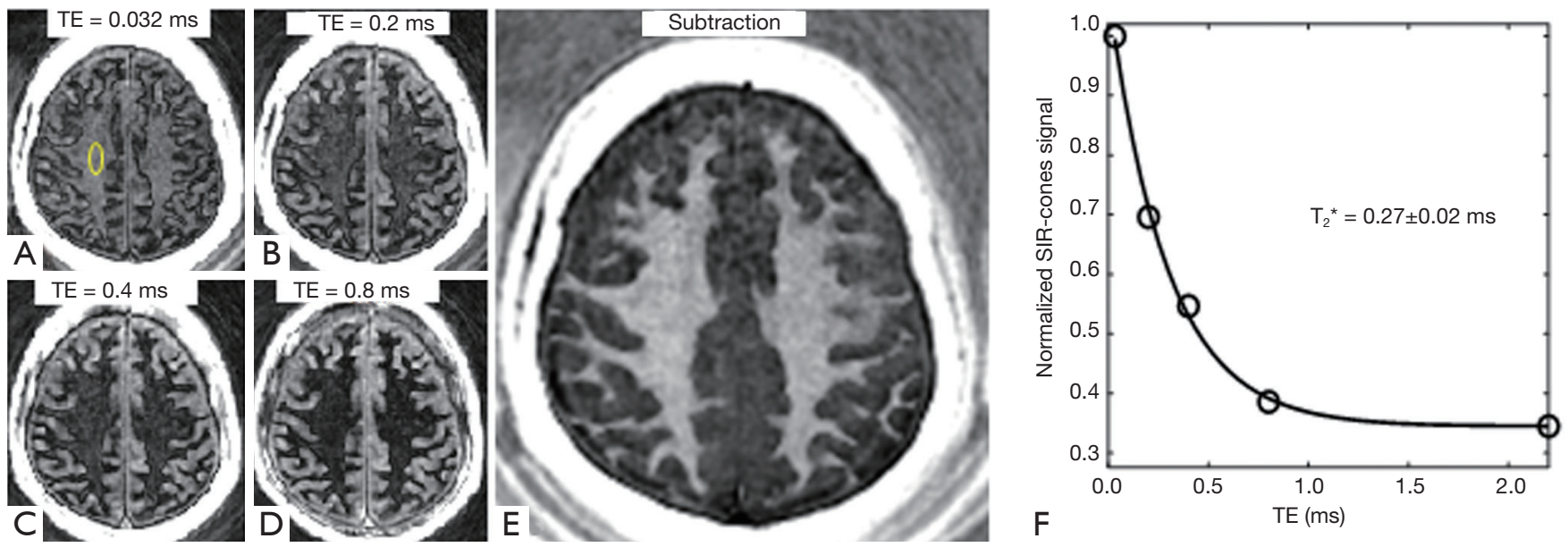

Figure 8 IR-UTE-Cones imaging of a 35-year-old volunteer with TEs of 0.032 (A), 0.2 (B), 0.4 ms (C), 0.8 ms (D), subtraction (E) (A minus D), and exponential fitting for a yellow oval ROI in (A), which shows a short $\mathrm{T}_{2}{ }^{*}$ of $0.27 \pm 0.02 \mathrm{~ms}(\mathrm{G})$. This in vivo myelin $\mathrm{T}_{2}{ }^{*}$ is very close to the $\mathrm{T}_{2}{ }^{*}$ of myelin-D2O paste as well as the $\mathrm{T}_{2}{ }^{*}$ of intact myelin vesicles, consistent with selective imaging of myelin using the $3 \mathrm{D}$ IR-UTE-Cones sequence at 3T. Adapted from (31), with permission.

near zero if an appropriate TI had been chosen to invert and null long $T_{2}$ white matter signal. The bright signal in Figure $7 B$ shows that an inaccurate TI was chosen. This might lead to significant residual signal from water protons (even at a TE of $1.2 \mathrm{~ms}, \mathrm{WM}_{\mathrm{L}}$ might have decayed to some degree, although much less than myelin), but the signal change in $W_{\mathrm{L}}$ might be even more than that of $\mathrm{WM}_{\mathrm{S}}$ as myelin has a far lower proton density. Therefore, the dual-echo subtraction image (Figure $7 C$ ) probably contains a significant amount of signal from water. BIcomponent $\mathrm{T}_{2}{ }^{*}$ analysis from IR-UTE imaging with a series of TEs would help determine how much water contamination was present in their study (or this could be done with $\mathrm{T}_{2}{ }^{*}$ spectra from an IR-UTE based FID of a pure white matter sample).

\section{Validation with healthy volunteer studies}

Figure 8 shows 3D IR-UTE-Cones imaging of a 35-year-old healthy volunteer using a clinical whole-body $3 \mathrm{~T}$ scanner (MR750, GE Healthcare, Milwaukee, WI, USA) (31). The longitudinal magnetization of $\mathrm{WM}_{\mathrm{L}}$ is inverted and nulled as evidenced by the near zero signal at a TE of $0.8 \mathrm{~ms}$. Subtraction of the TE $=0.8 \mathrm{~ms}$ image from the $1^{\text {st }}$ one provides selective imaging of myelin in white matter, as further confirmed by the short $\mathrm{T}_{2}{ }^{*} \mathrm{~s}$ of $0.2-0.3 \mathrm{~ms}$ measured in white matter, which are close to the $\mathrm{T}_{2}{ }^{*} \mathrm{~s}$ of myelin phantoms (Figure 2) and porcine myelin vesicles (Figure 4).

\section{Validation with MS patient studies}

Figure 9 shows MP-RAGE, T 2 -FLAIR, and 3D IR-UTECones imaging of two representative MS patients (both 62 years old, one female, one male) (31). Similar to our observations in the ex vivo study, myelin signal loss in MS lesion regions can be seen on the 3D IR-UTE-Cones images in both patients, demonstrating that the $3 \mathrm{D}$ IRUTE-Cones sequence can directly show myelin loss in MS lesions in vivo. Mean $T_{2}{ }^{*}$ s for normal appearing white matter (NAWM) in MS patients on subtraction images typically are around $0.3 \mathrm{~ms}$, similar to the $\mathrm{T}_{2}{ }^{*}$ values of myelin in phantoms, myelin vesicles, and healthy volunteers.

\section{Other myelin imaging techniques}

\section{Long $T_{2}$ suppressed UTE imaging of myelin}

UTE imaging with long $T_{2}$ saturation has been proposed for myelin imaging in vivo (35). The primary design parameter is the time $\times$ bandwidth product (TBW), which is proportional to the spectral profile sharpness. Simple rectangular pulses, single-band pulses and dual-band pulses have been investigated. Figure 10 shows brain UTE images with and without long- $\mathrm{T}_{2}$ suppression in a healthy volunteer. The use of long- $T_{2}$ suppression pulses reveals a short $T_{2}$ component in white matter that is completely obscured without suppression. This component is believed to be associated with myelin, as has previously been 

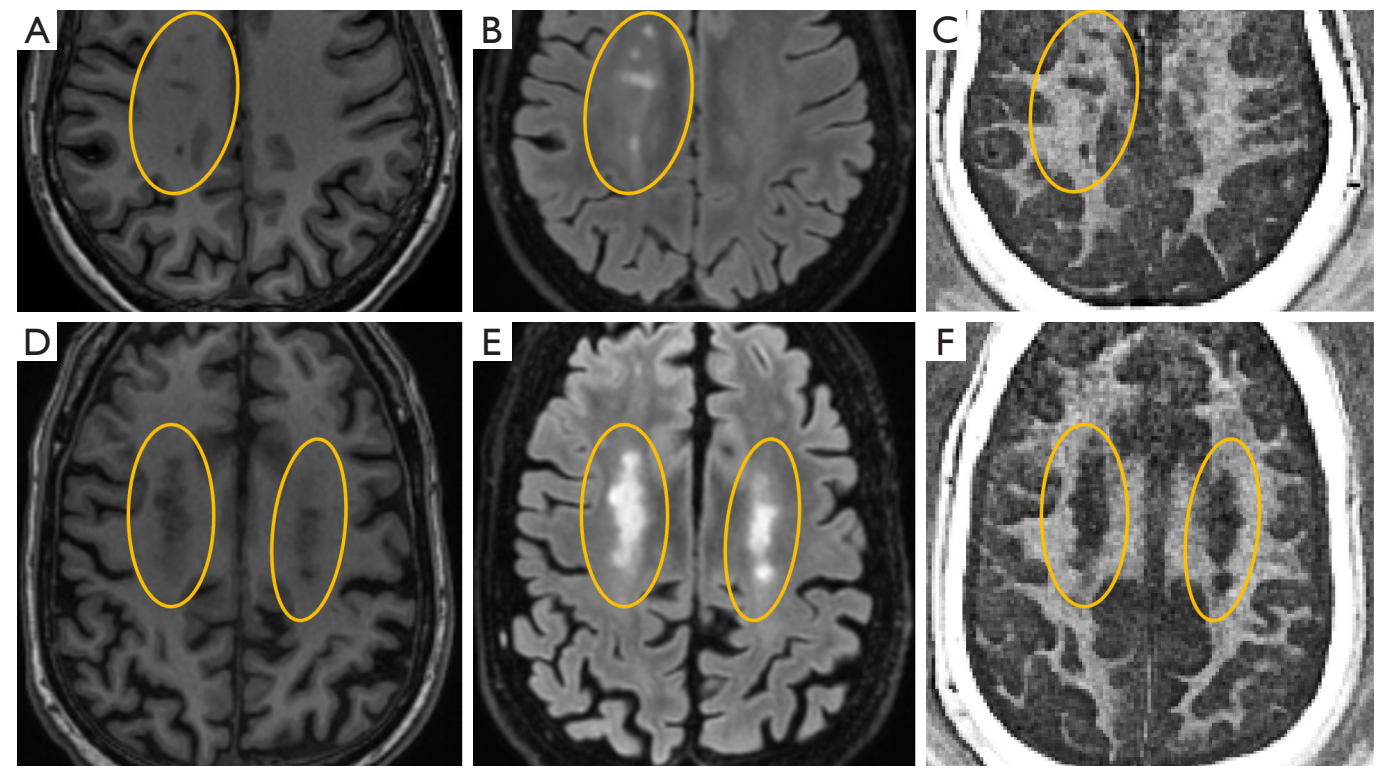

Figure 9 Clinical MP-RAGE (A), T ${ }_{2}$-FLAIR (B) and 3D IR-UTE-Cones (C) images of a 62yo female MS patient, and MP-RAGE (D), T ${ }_{2}-$ FLAIR (E), and 3D IR-UTE-Cones (F) images of a 62 yo male MS patient. MS lesions are highlighted with orange ovals. Myelin loss is depicted on the subtracted 3D IR-UTE-Cones images (C and F) with high contrast. Adapted from (31), with permission.

suggested by its loss in patients with MS (18). Short $T_{2}$ tissue components in the falx cerebri (long, thin arrows in Figure 10) also have significantly improved visualization with long- $T_{2}$ suppression. A major problem associated with long- $T_{2}$ suppressed UTE imaging of myelin is its high sensitivity to both $\mathrm{B}_{1}$ and $\mathrm{B}_{0}$ field inhomogeneities. $B_{1}$ inhomogeneity produces spatially dependent long- $T_{2}$ suppression, leading to significant water signal contamination in regions with a lower $\mathrm{B}_{1} \cdot \mathrm{B}_{0}$ inhomogeneity also reduces the efficiency of long- $T_{2}$ suppression leading to significant water signal contamination.

\section{ZTE with echo subtraction for myelin imaging}

More recently, Weiger et al. employed a novel technique called hybrid filling (HYFI) for improved scan efficiency in zero echo time (ZTE) imaging with large dead-time gaps, for direct imaging of myelin (36). They acquired two HYFI datasets with TEs of 15 and $503 \mu$ s, respectively. They used an extremely high slew rate and gradient amplitude (600 m T/m/ms and $200 \mathrm{mT} / \mathrm{m}$ respectively). Echo subtraction was used to suppress long $\mathrm{T}_{2}$ signals, creating high contrast for myelin. Figure 11 shows their in vivo myelin images. As demonstrated in their abstract, ZTE/ PETRA type encoding can shorten the effective TE, which can be advantageous in imaging myelin protons with very short $\mathrm{T}_{2}{ }^{*} \mathrm{~s}$, especially when combined with an extremely high slew rate and high gradient amplitude. However, it is very challenging to achieve selective myelin imaging by direct subtraction of two UTE images, as the signal from long $T_{2}$ water is far higher than that from myelin in white matter of the brain. Imperfect long- $T_{2}$ suppression may lead to significant water contamination, let alone not using long- $\mathrm{T}_{2}$ suppression at all as in this study. Dual-echo UTE with echo subtraction (with no long- $\mathrm{T}_{2}$ suppression pulses) and UTE with long- $T_{2}$ saturation (which is very sensitive to $B_{1}$ inhomogeneity, and may result in imperfect water suppression) both have problems in generating myelin selective images. Adiabatic IR-UTE appears to be a better approach but the choice of TI is of critical importance, as an incorrect choice of TI may lead to significant water contamination, as shown in Figure 7.

\section{Dual echo UTE imaging with complex echo subtraction for myelin imaging}

In dual-echo IR-UTE imaging of myelin, complex echo subtraction may further improve the myelin contrast generated by magnitude image subtraction by removing the phase error caused by the initial phase offset and phase evolution due to field inhomogeneity (37). In contrast to magnitude subtraction, complex subtraction is not 

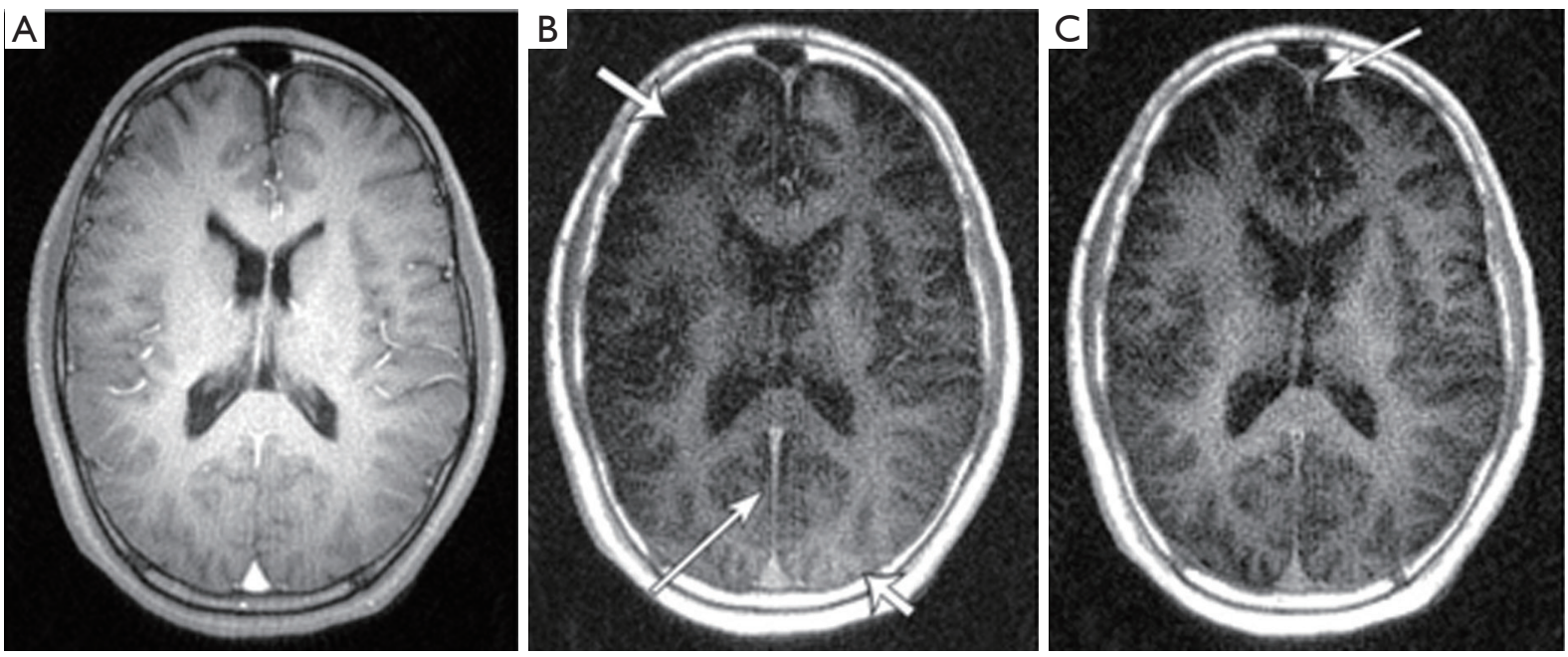

Figure 10 UTE Brain images with and without long- $T_{2}$ suppression. (A) Unsuppressed image. (B) 16-ms rectangular suppression pulse. (C) 40-ms TBW =2.4 single-band suppression pulse. With the suppression pulses, short- $\mathrm{T}_{2}$ components in the white matter, probably from myelin are visible (as is the falx cerebri, long, thin arrows). The rectangular pulse suppression in (B) is variable across the slice (shorter, wider arrows), while the single-band pulse produces more consistent suppression in (C). From (35), with permission.

affected by the initial phase of the myelin and white matter signals. Complex subtraction requires one additional step to correct for the phase offset, $\phi$, which can be caused by phase evolution during the RF pulse or data acquisition, as well as by imperfect image reconstruction (38), and a second step to correct for the phase error induced by $\mathrm{B}_{0}$ field inhomogeneity. Figure 12 shows 3D IR-UTE-Cones imaging of a 29-year-old volunteer using magnitude and complex subtraction, respectively, with the latter providing improved contrast and better detection of fine myelin structures.

\section{Dual Echo Sliding Inversion REcovery (DESIRE) UTE imaging of myelin}

$\mathrm{T}_{1}$ values of $W M_{\mathrm{L}} / \mathrm{GM}_{\mathrm{L}}$ may vary in different $\mathrm{WM} /$ GM regions of the brain in the same person and between subjects. $T_{1}$ variations in different regions of the same subject and errors in $T_{1}$ measurement both lead to imperfect nulling of $W M_{L}$ and/or $G_{L}$ if a single value of TI is used for the whole brain. DESIRE-UTE can be used to address this problem and obtain accurate and robust long- $\mathrm{T}_{2}$ suppression by obtaining a range of TIs for each voxel and using the one that best nulls long- $\mathrm{T}_{2}$ signals (Figure 13) $(34,39)$. The key components are: First, a sufficient number of continuous image spokes is used to cover a wide range of TIs including all potential nulling points for $W_{\mathrm{L}}$ with its different $T_{1} s$; Second, each group of spokes needs to cover k-space uniformly with random gradient encoding; Third, dual echo images need to be acquired with each single spoke to allow image subtraction; Fourth, advanced image reconstruction techniques such as parallel imaging and/or compressed sensing (CS) are necessary to reduce undersampling artifacts (40). For each pixel in the brain, the best TI for nulling is the one which results in the lowest signal on the $2^{\text {nd }}$ echo image.

Figure 14 shows 3D DESIRE-UTE imaging of a 44-year-old volunteer. Data corresponding to 71 equally spaced TIs are collected per IR. A sliding window reconstruction scheme shown in Figure 13 is employed to generate images with a broad range of potential nulling points to suppress $W M_{L}$ and $G M_{L}$ with their different $T_{1} s$. Volumetric myelin imaging can be achieved in WM (nulling of $W_{\mathrm{L}}$ ) and in GM (nulling of $\mathrm{GM}_{\mathrm{L}}$ ), respectively. From Ref. 33, with permission.

Figure 15 shows MP-RAGE, $\mathrm{T}_{2}$-FLAIR, and DESIRE UTE imaging of a 45-year-old patient. DESIRE UTE provides more efficient water suppression, especially for regions with varying $T_{1} s$ due to pathology, thus providing robust imaging of myelin. Lesions are depicted with excellent contrast. The normalized DESIRE signal is $0.76 \pm 0.11$ for NAWM and $0.19 \pm 0.10$ for MS lesions consistent with significant myelin loss. Myelin density maps can be generated by comparing the DESIRE UTE signal with that of a phantom, after correction for $T_{1}, T_{2}{ }^{*}$ and coil sensitivity effects. 


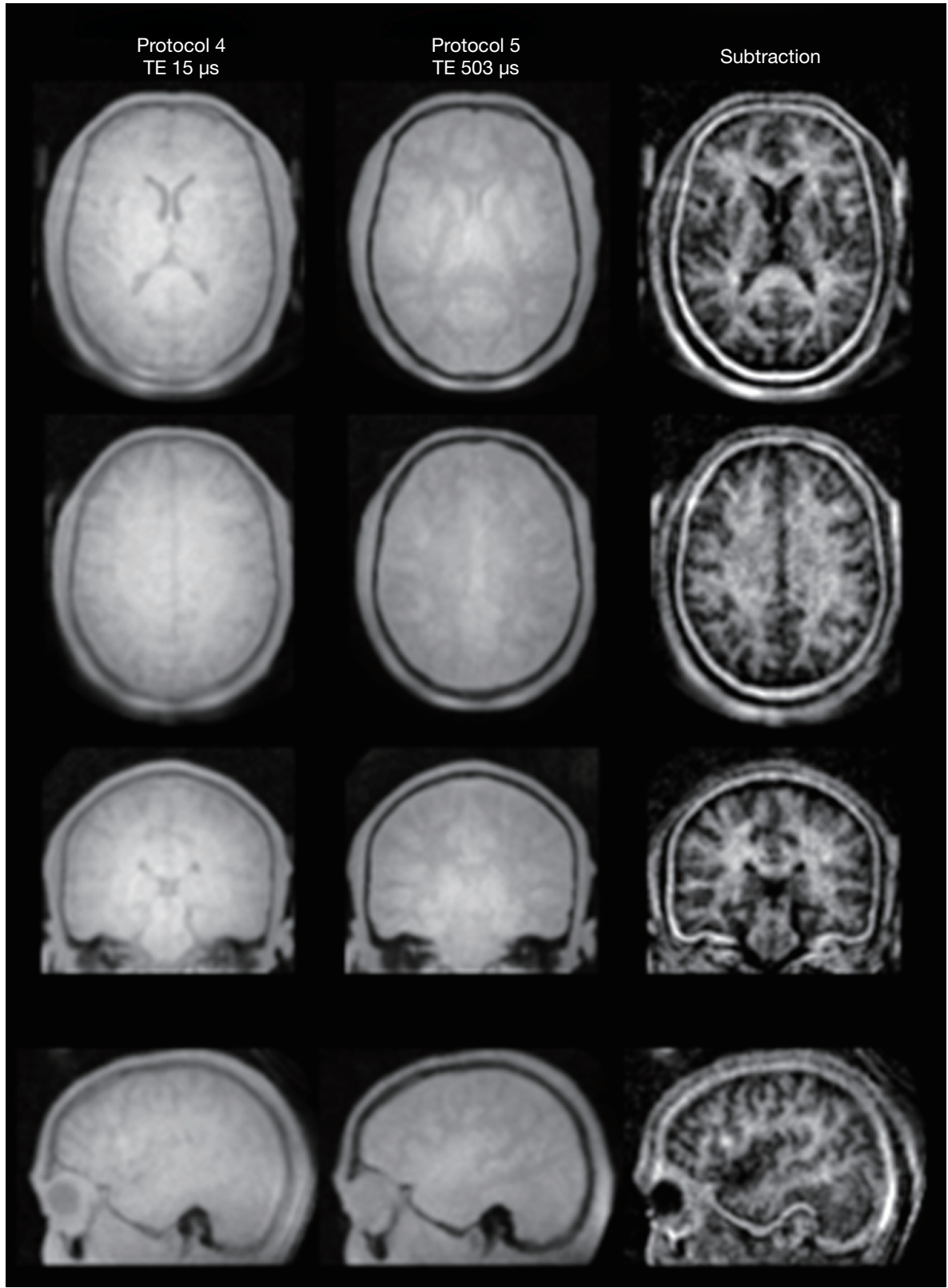

Figure 11 In vivo imaging of the ultrashort $\mathrm{T}_{2}$ components in the human brain. Selected views from a $3 \mathrm{D}$ isotropic data set are shown. Images at the shortest TE of $15 \mu$ s show little WM-GW contrast (Protocol 4). Increasing TE leads to reduced signal in WM (Protocol 5). Subtraction of the $2^{\text {nd }}$ from the $1^{\text {st }}$ data set largely removes the long- $T_{2}$ signal, showing predominantly ultrashort $\mathrm{T}_{2}$ components with clear WM-GW contrast (subtraction column). From (36), with permission.

\section{UTE magnetization transfer (UTE-MT) imaging of myelin}

The combination of UTE and magnetization transfer (UTE-MT) has also been proposed for myelin imaging and quantification (41). In a recent study by Guglielmetti et al.,
UTE-MT data with an ultrashort TE of $76 \mu$ s and short TE MT (STE-MT) data with a longer TE of $3 \mathrm{~ms}$ were used to evaluate spatial and temporal changes in brain myelin content in a cuprizone mouse model of MS on a clinical 7T scanner. Both UTE-MT ratio (UTE-MTR) and STE- 

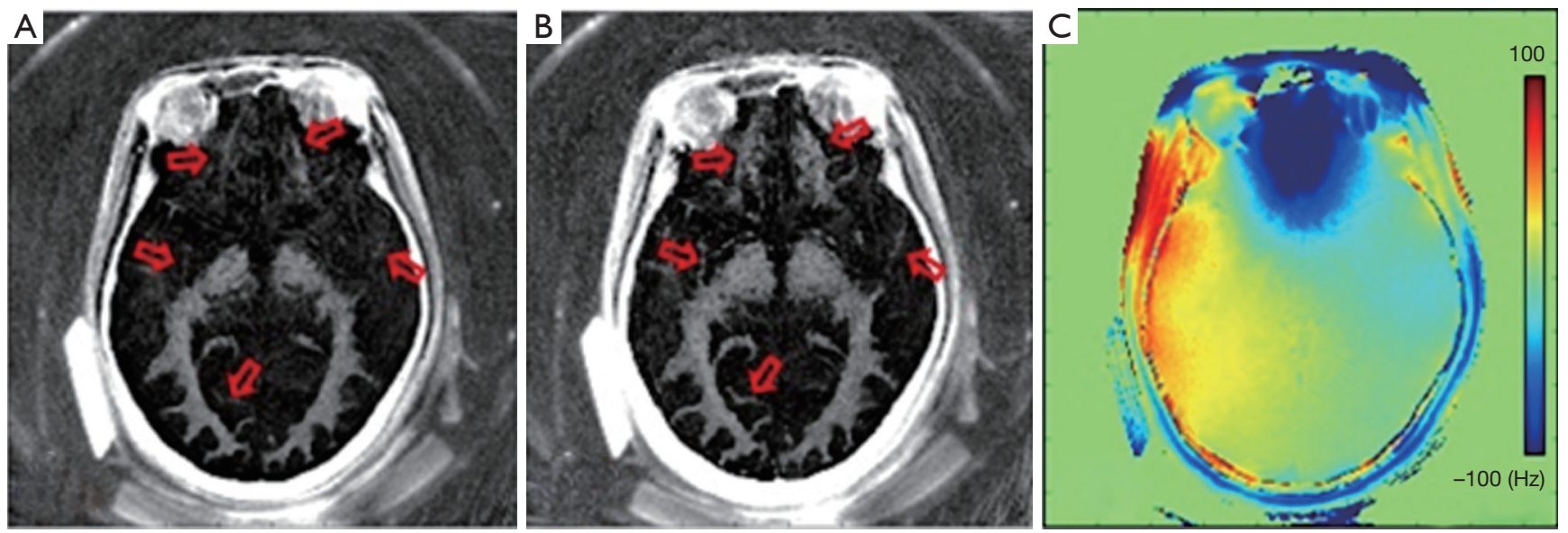

Figure 12 3D IR-UTE-Cones imaging of a 29-year-old volunteer using magnitude (A) and complex (B) subtraction, and the field map (C). The complex subtraction provides improved myelin detection, especially for fine structures and in regions with strong off-resonance effects (arrows). Adapted from (37), with permission.

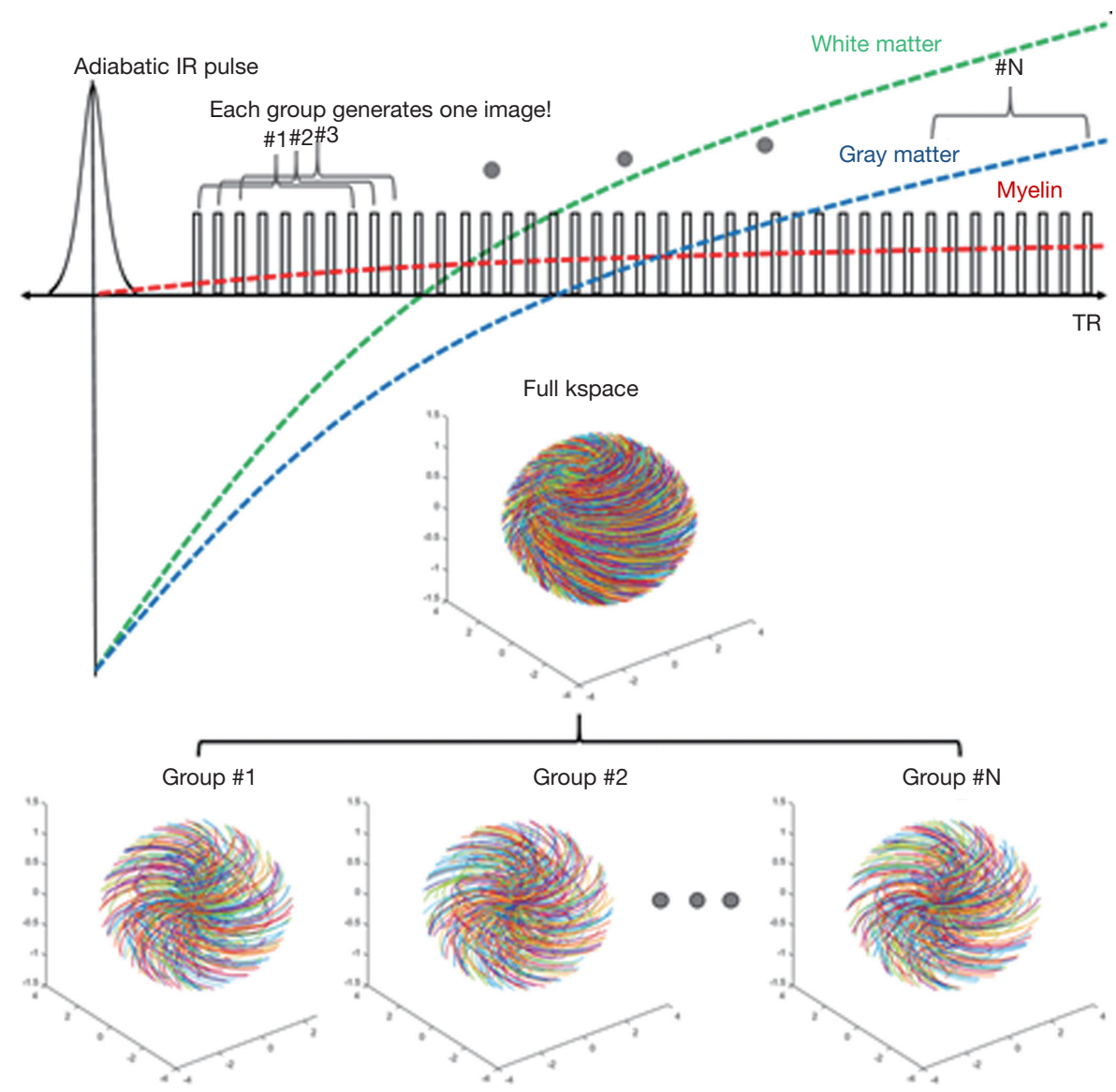

Figure 13 DESIRE contrast mechanism: a series of dual echo UTE spokes are acquired after each IR, a sliding window reconstruction is employed to generate one image per spoke, with up to 71 images, each of which has a different TI. The longitudinal magnetizations of $\mathrm{WM}_{\mathrm{L}}$ can be nulled with an appropriate TI for each voxel, thus allowing robust selective imaging of myelin in WM of the whole brain in different subjects in spite of differences in $\mathrm{T}_{1}$ of $W \mathrm{M}_{\mathrm{L}}$. 


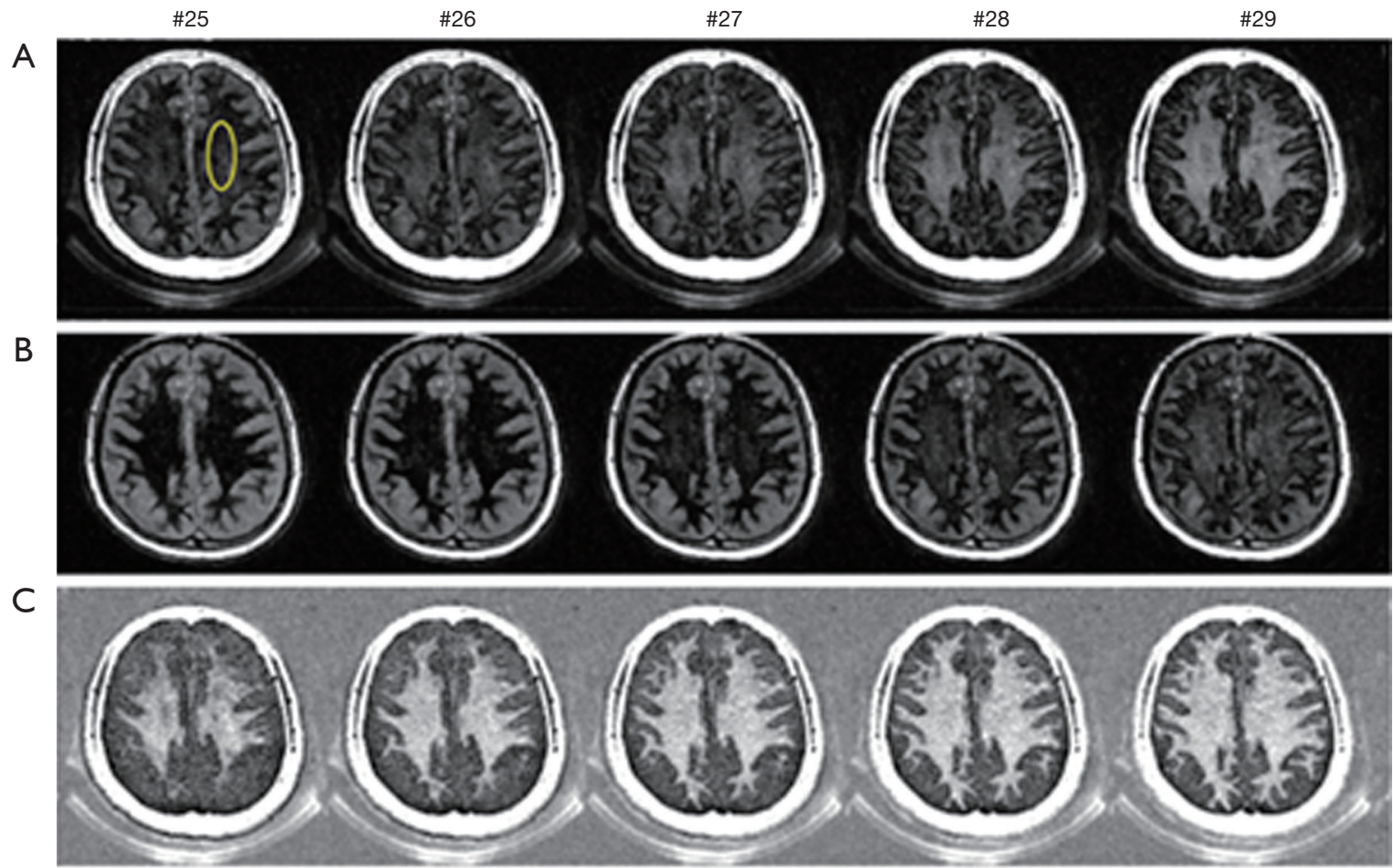

D

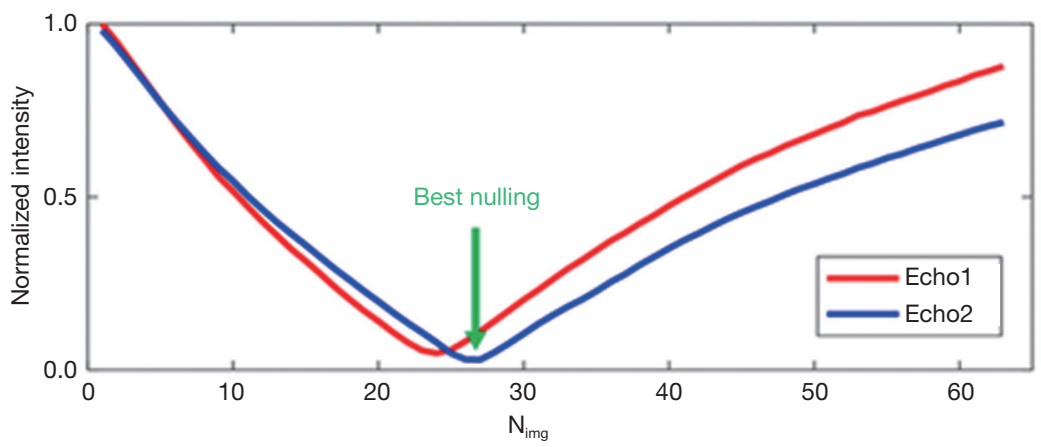

Figure 14 DESIRE-UTE imaging and echo-subtraction at the TIs close to the long T2 nulling point. (A) Five out of 71 DESIRE reconstructed sequential sets of 3D IR-UTE-Cones images of the brain in a 44-year-old volunteer with a TR of $1 \mathrm{~s}$, TEs of $0.03 \mathrm{~ms}$ (A) and $2.2 \mathrm{~ms}$ (B), the corresponding subtraction images (C), and the signal intensity $v$ s. sliding window reconstructed images for an ROI in WM (A). Selective myelin imaging is achieved when the 2 nd echo shows a null value for WML (arrow in D). From (34), with permission.

MT ratio (STE-MTR) values were significantly decreased in most white matter and gray matter regions during demyelination. However, only UTE-MTR detected cortical changes. UTE-MTR values remained lower than baseline values after remyelination in subcortical and cortical areas. Those results show that UTE-MT, but not STE-MT, detects long-lasting changes following a demyelinating event. UTE-MTR also shows much stronger correlation $\left(\mathrm{R}^{2}\right.$ $=0.71)$ than STE-MTR $\left(\mathrm{R}^{2}=0.48\right)$ with percent coverage of myelin basic protein immunostaining. No correlation was observed between UTE-MTR or STE-MTR and gliosis immunostaining. Those results suggest that UTE-MT methods may provide non-invasive longitudinal monitoring of demyelinated lesions, not only during disease progression but also in response to remyelinating therapies (41).

\section{Challenges in direct UTE MR imaging of myelin}

While extensive validation studies have been performed on direct MRI of myelin (21-28), there is ongoing debate 

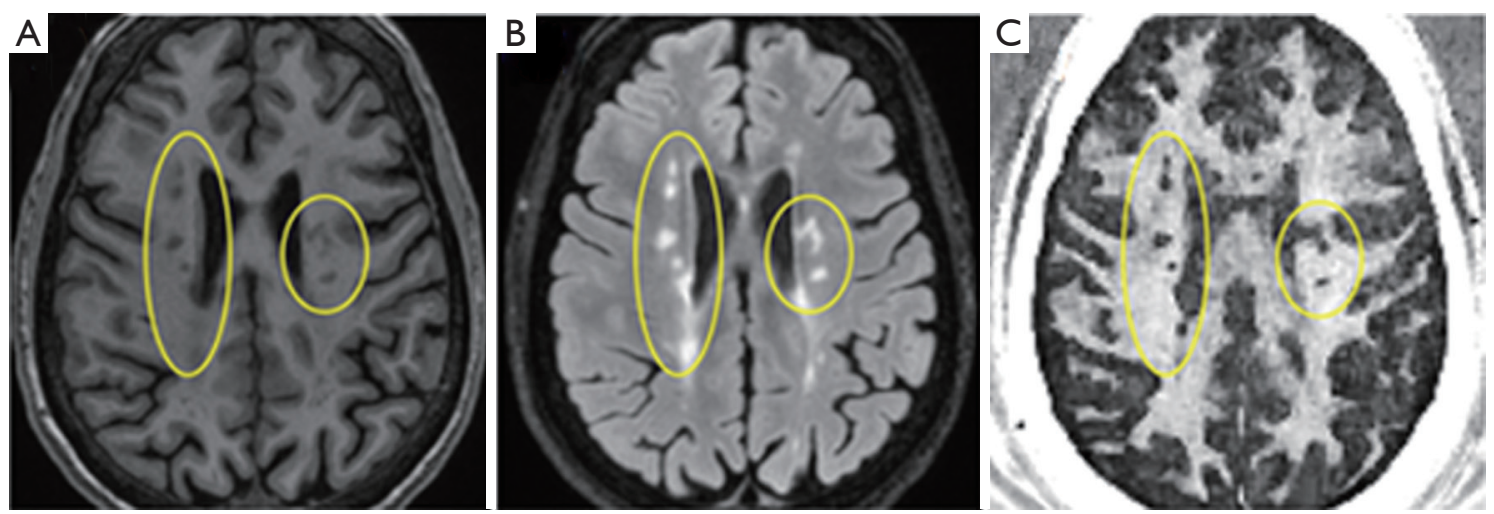

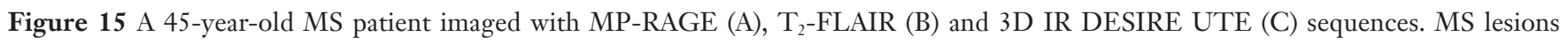
labeled with ellipses on the MP-RAGE and $\mathrm{T}_{2}$-FLAIR images are shown as signal voids on DESIRE images, corresponding to myelin loss. From (34), with permission.

regarding the signal sources in UTE imaging of myelin. A major critique is the measured $\mathrm{T}_{2}{ }^{*}$ of $\sim 0.3 \mathrm{~ms}$, which is much longer than the $T_{2}{ }^{*}$ of $\sim 0.05$ ms measured with high performance NMR spectrometers $(17,19,20)$. Broad line proton spectroscopy studies have shown that myelin is in a liquid-crystalline state (17). Multi-component fitting of spin echo (SE) decays of fixed human white matter samples has shown extremely short $T_{2}$ values of $\sim 50 \mu \mathrm{s}$ for myelin protons (42). NMR spectrometer studies have found ultrashort $T_{2}$ signals ( $50 \mu \mathrm{s}<\mathrm{T}_{2}<1 \mathrm{~ms}$ ) in myelinated nerve (19), and a wide distribution of $T_{2}{ }^{*}$ values ranging from $8 \mu$ s to $26 \mathrm{~ms}$ in the spinal cord (20). UTE sequences on clinical MR scanners are unlikely to detect the $\sim 50 \mu \mathrm{s}$ component of myelin, but can be expected to detect the longer $\mathrm{T}_{2}{ }^{*}$ components. Another possibility is that myelin has a bilayer structure and methylene protons on myelin lipids may be subject to the magic angle effect between the bilayers and the magnetic field, leading to the longer $T_{2}{ }^{*}$ measured with UTE sequences on clinical systems. More research is still needed to explain the $T_{2}{ }^{*}$ values measured with IR-UTE sequences on clinical MR scanners.

Another major critique is about the well-defined edges on echo subtracted IR-UTE images, which appear inconsistent with the fact that myelin has an extremely short $\mathrm{T}_{2}{ }^{*}$ and should therefore be subject to severe blurring. However, echo subtracted IR-UTE images typically display sharp edges as shown in Figures 5,6,8,9,12,14,15. This obvious contradiction can be explained by the contrast mechanism described in Figure 1. Briefly, in dual-echo IR-UTE imaging the gray matter region has a positive longitudinal magnetization for myelin but negative longitudinal magnetization for $\mathrm{GM}_{\mathrm{L}}$, leading to signal cancellation in the initial image. However, no such magnetization cancellation exists in the $2^{\text {nd }}$ echo image due to the fast signal decay in myelin. Meanwhile, white matter has only a positive longitudinal magnetization of myelin in the $1^{\text {st }}$ image (assuming $W_{\mathrm{L}}$ is well suppressed) and pure noise in the $2^{\text {nd }}$ echo image. As a result, echo subtraction leads to positive myelin signal in white matter but negative signal in gray matter, creating very high contrast on echosubtracted IR-UTE images. The end result is an apparently sharp boundary between myelin in white matter and gray matter, although the myelin signal is subject to significant blurring due to the use of a relatively long sampling window compared to its ultrashort $\mathrm{T}_{2}{ }^{*}$.

Recently Harkins and Does conducted a feasibility study on in vivo myelin imaging using adiabatic inversion recovery (AIR) to null signal of a single $T_{1}$, or multiple adiabatic inversion recovery (MAIR) to null signal over a range of $\mathrm{T}_{1} \mathrm{~s}$ (43). They found that AIR-UTE showed contrast in white matter, while MAIR-UTE showed no signal but pure noise in white matter of the brain in vivo, as shown in Figure 16. The AIR image shows signal in various locations, including white matter regions, the skull bone, and fat around the skull. The MAIR image also shows relatively large signals from bone and fat, but no signal from white matter regions. They concluded that the AIR-UTE white matter signals were unsuppressed water signals and that the proton signals of myelin decayed too quickly to be observed by UTE MRI (43). A major problem with this study is the lack of recognition of signal cancellation between uninverted myelin longitudinal magnetization and inverted 

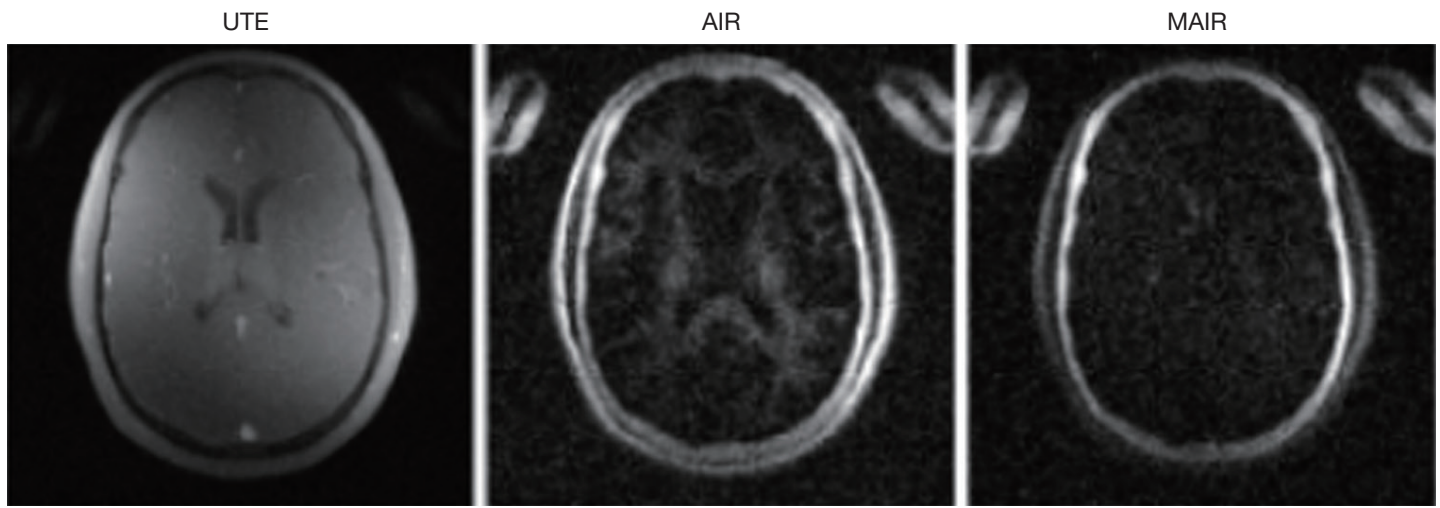

Figure 16 UTE, AIR, and MAIR brain images acquired from a healthy volunteer. AIR and MAIR images both contain ultrashort $\mathrm{T}_{2}$ signals in the skull and elements of the RF coil. The low intensity signals within white matter regions of the brain are present in the AIR image but absent in MAIR image, and, therefore, do not appear to originate from myelin. From (42), with permission.

water longitudinal magnetization in both white and gray matter. Imperfect combinations of TR and TI may lead to water-myelin cancellation and so no detectable signal. More research is still needed to understand the factors contributing to the absence of signal from white matter in MAIR imaging.

Another challenge is to improve the imaging of myelin in gray matter, as gray matter has a much lower myelin content than white matter. The lower water content of white matter $(72 \%)$ compared to gray matter $(82 \%)$ is largely due to the higher myelin content of white matter (44). UTE bicomponent analysis of native ovine white matter suggests that only $\sim 8 \%$ of the total UTE signal has a short $\mathrm{T}_{2}{ }^{*}$ of $\sim 0.3 \mathrm{~ms}$ (27). The very low myelin signal fraction arises from the fact that myelin density is low in white matter, and its $T_{2}{ }^{*}$ is extremely short. This leads to low efficiency in exciting myelin magnetization, and significant blurring during UTE data acquisition especially using clinical whole-body scanners with their relatively low slew rates and gradient strength. The myelin signal fraction is much lower in gray matter than white matter. Therefore, there is more need for efficient long- $T_{2}$ suppression using more advanced imaging techniques such as DESIRE. A more recently developed technique called Short Tr Adiabatic Inversion Recovery (STAIR) $(45,46)$ suppresses signals with a broad range of $\mathrm{T}_{1} \mathrm{~s}$ using a short $\mathrm{TR}$ with IR-UTE imaging. The STAIR-UTE technique may allow more robust imaging of myelin in both white and gray matter. The ultrahigh slew rate and gradient amplitude used in the Weiger study is also likely to help improve the sensitivity in IR-UTE imaging of myelin in vivo (36).
Water contamination is by far the most challenging problem in direct imaging of myelin on clinical MR scanners. As mentioned earlier, the spatial saturation based approach (35) or simple echo subtraction (36) may be subject to significant water contamination. Dual echo IR-UTE imaging followed by echo subtraction provides selective myelin imaging, on the condition that an appropriate TI is selected to invert and null $W_{\mathrm{L}}$. An inaccurate TI leads to significant water signal contamination (20). Even a slightly longer or shorter TI than the optimum one for nulling may lead to significant water signal contamination, especially considering the much lower proton density and much shorter $T_{2}{ }^{*}$ of myelin relative to water. Imperfect nulling compromises the specificity and accuracy of myelin imaging. Further, the optimal nulling point $\left(\mathrm{TI}_{\text {null }}\right)$ for $\mathrm{WM}_{\mathrm{L}}$ may be difficult to determine. The $T_{1}$ of normal $W_{L}$ varies in different brain regions and between individuals $(47,48)$. A wide range of $T_{1}$ values have been reported (690-1,100 ms) for WM at $3 \mathrm{~T}(49,50)$. Moreover, the $\mathrm{T}_{1}$ of $\mathrm{WM}_{\mathrm{L}}$ may change with disease involving white matter. $\mathrm{T}_{1}$ measurement is also time-consuming, which is another limitation in clinical applications. As a result, the dual-echo IR-UTE with echo subtraction technique will fail in those cases, leading to significant water signal contamination and thus errors in myelin quantification. Myelin water is another potential contaminator since a variety of recent studies have demonstrated that myelin water has a relatively short $\mathrm{T}_{2}(\sim 10-50 \mathrm{~ms})$ and $\mathrm{T}_{2}{ }^{*}(\sim 3-10 \mathrm{~ms})$ when compared to the longer $T_{2}$ components in white matter (51-53). The DESIRE-UTE technique deals with these challenges by generating a series of TIs (34), to provide an optimal single 
TI for nulling $\mathrm{WM}_{\mathrm{L}}$ in each voxel and thus nulling of $\mathrm{WM}_{\mathrm{L}}$ throughout the brain irrespective of its $\mathrm{T}_{1}$. STAIR-UTE uses a shorter TR to increase the efficiency of suppressing long $T_{2}$ components with a broad range of $T_{1}$ s. The advantages of direct myelin imaging over myelin water imaging remain to be investigated. More research is needed in this area.

Finally, it is important to note that remyelinationenhancing therapies for MS patients are being developed (54). Preclinical research has identified multiple targets affecting remyelination in animal models of MS (55-58). Early stage human clinical trials of drugs affecting myelination have started, including Natalizumab (NCT01416181), Fingolimod (NCT00731692), Alemtuzumab (NCT01307332, NCT01395316), LINGO-1 (NCT01244139) and rHIgM22 (NCT01803867). However, the lack of a robust biomarker to monitor the effectiveness of these drugs in enhancing remyelination highlights the importance of developing direct imaging of myelin using UTE or similar sequences on clinical whole-body MR systems.

\section{Conclusions}

A series of studies have demonstrated that myelin is detectable and can be imaged with UTE type sequences using clinical whole-body scanners. Myelin in white matter of the brain can be selectively imaged with the IR-UTE sequence, where an adiabatic inversion pulse is used to invert and null signals from $W M_{L}$, the longer $T_{2}$ components in white matter. The residual longer $T_{2}$ signal from $\mathrm{GM}_{\mathrm{L}}$ can be suppressed via dual echo acquisition and echo subtraction. More recently developed techniques, such as the DESIRE UTE and STAIR-UTE are expected to provide more robust morphological imaging of myelin, although quantitative myelin density mapping remains to be established.

\section{Acknowledgments}

Funding: This study was supported by the National Institutes of Health (R01NS092650).

\section{Footnote}

Conflicts of Interest: All authors have completed the ICMJE uniform disclosure form (available at http://dx.doi. org/10.21037/qims-20-541). JD serves as an unpaid editorial board member of Quantitative Imaging in Medicine and Surgery. The other authors have no conflicts of interest to declare.

Open Access Statement: This is an Open Access article distributed in accordance with the Creative Commons Attribution-NonCommercial-NoDerivs 4.0 International License (CC BY-NC-ND 4.0), which permits the noncommercial replication and distribution of the article with the strict proviso that no changes or edits are made and the original work is properly cited (including links to both the formal publication through the relevant DOI and the license). See: https://creativecommons.org/licenses/by-nc-nd/4.0/.

\section{References}

1. van der Knaap MS, Valk J. Magnetic Resonance of Myelination and Myelin Disorders. Berlin: Springer, 2005;1-19.

2. Paty DW, Li DK, Oger JJ, Kastrukoff L, Koopmans $\mathrm{R}$, Tanton E, Zhao GJ. Magnetic resonance imaging in the evaluation of clinical trials in multiple sclerosis. Ann Neurol 1994;36:S95-6.

3. Miller DH, Barkhof F, Nauta JJP. Gadolinium enhancement increases the sensitivity of MR in detecting disease activity in multiple sclerosis. Brain 1993;116:1077-94.

4. Filippi M, Inglese M. Overview of diffusion-weighted magnetic resonance studies in multiple sclerosis. J Neurol Sci 2001;186:S37-43.

5. Schmierer K, Scaravilli F, Altmann DR, Barker GJ, Miller DH. Magnetization transfer ratio and myelin in postmortem multiple sclerosis brain. Ann Neurol 2004;56:407-15.

6. Filippi M, Rocca MA. MR imaging of multiple sclerosis. Radiology 2011; 259:659-81.

7. Filippi M, Rocca MA. Conventional MRI in multiple sclerosis. J Neuroimaging 2007;17:3S-9S.

8. Bakshi R, Thompson AJ, Rocca MA, Pellitier D, Dousset V, Barkhof F, Inglese M, Guttman CR, Horsfield MA, FIllipi M. MRI in multiple sclerosis: current status and future prospects. Lancet Neurol 2008;7:615-25.

9. Li DK, Held U, Petkau J, Daumer M, Barkhof F, Fazekas F, Frank JA, Kappos L, Miller DH, Simon JH, Wolnsky JS, Fillippi M. MRI T2 lesion burden in multiple sclerosis: a plateauing relationship with clinical disability. Neurology 2006;66:1384-9.

10. Nijeholt GJ, van Walderveen MA, Casteljns JA, van Waesberghe JH, Polman C, Schelens P, Rosier PF, Jorgen 
PJ, Barkhof F. Brain and spinal cord abnormalities in multiple sclerosis, correlation between MRI parameters, clinical subtypes and symptoms. Brain 1998;121:687-97.

11. Charil A, Zijdenbos AP, Taylor J, Boelman C, Worseley KJ, Evans AC, Dagher A. Statistical mapping analysis of lesion location and neurological disability in multiple sclerosis: application to 452 patient data sets. Neuroimage 2003;19:532-44.

12. Kappos L, Moeri D, Radue EW, Schoetzau A, Schweikert K, Barkhof F, Miller D, Guttmann CRG, Weiner HL, Gasperini C, Filippi M, for the Gadolinium MRI metaanalysis group. Lancet 1999;353:964-9.

13. Rovaris M, Judica E, Sastre-Garriga J, Rovira A, Sormani MP, Benedetti B, Korteweg T, Stefano ND, Khaleeli Z, Montalban X, Barkhof F, Miller DH, Polman C, Thompson AJ, Filippi M. Large-scale, multicenter, quantitative MRI study of brain and cord damage in primary progressive multiple sclerosis. Multiple Sclerosis 2008;14:455-64.

14. Schmierer K, Claudia AM, Wheeler-Kingshott, Tozer DJ, Boulby PA, Parkes HG, Yousry TA, Scaravilli F, Barker GJ, Tofts PS, Miller DH. Quantitative magnetic resonance of postmortem multiple sclerosis brain before and after fixation. Magn Reson Med 2008;59:268-77.

15. Newcombe J, Hawkins CP, Henderson CL, Patel HA, Woodroofe MN, Hayes GM, Cuzner LM, MacManus D, du Boulay EP, McDonald WI. Histopathology of multiple sclerosis lesions detected by magnetic resonance imaging in unfixed postmortem central nervous system tissue. Brain 1991;114:1013-23.

16. Gareau PJ, Rutt BK, Karlik SJ, Mitchell JR. Magnetization transfer and multicomponent $\mathrm{T} 2$ relaxation measurements with histopathologic correlation in an experimental model of MS. J Magn Reson Imaging 2000;11:586-95.

17. Lecar H, Ehrenstein G, Stillman I. Detection of molecular motion in lyophilized myelin by nuclear magnetic resonance. Biophys J 1971;11:140-5.

18. Waldman A, Rees JH, Brock CS, Robson MD, Gatehouse PD, Bydder GM. MRI of the brain with ultra-short echo time pulse sequences. Neuroradiology 2003;45:887-92.

19. Horch RA, Gore JC, Does MD. Origins of the ultrashort T2 1H NMR signals in myelinated nerve: a direct measure of myelin content? Magn Reson Med 2011;66:24-31.

20. Wilhelm MJ, Ong HH, Wehrli SL, Li C, Tsai PH, Hackney DB, Wehrli FW. Direct magnetic resonance detection of myelin and prospects for quantitative imaging of myelin density. Proc Natl Acad Sci USA 2012;109:9605-10.
21. Du J, Ma G, Li S, Carl M, Szeverenyi N, VandenBerg S, Corey-Bloom J, Bydder GM. Ultrashort TE echo time (UTE) magnetic resonance imaging of the short T2 components in white matter of the brain using a clinical 3T scanner. NeuroImage 2014;87:32-41.

22. Du J, Sheth V, He Q, Carl M, Chen J, Corey-Bloom J, Bydder GM, Du J. Measurement of T1 of the ultrashort T2* components in white matter of the brain at $3 \mathrm{~T}$. PLoS One 2014;9:e103296.

23. Sheth V, Shao H, Chen J, VandenBerg S, Corey-Bloom J, Bydder GM, Du J. Magnetic resonance imaging of myelin using ultrashort echo time (UTE) pulse sequence: phantom, specimen, volunteers and multiple sclerosis patient studies. NeuroImage 2016;136:37-44.

24. Sheth VR, Fan S, He Q, Ma Y, Annesse J, Switzer R, Corey-Bloom J, Bydder GM, Du J. Inversion recovery UTE magnetic resonance imaging: a method for simultaneous direct detection of myelin and high signal demonstration of iron deposition in the brain-a feasibility study. Magn Reson Imaging 2017;38:87-94.

25. He Q, Ma M, Shao H, Sheth V, Bydder G, Du J. Direct magnitude and phase imaging of myelin using ultrashort echo time (UTE) pulse sequences: a feasibility study. Magn Reson Imaging 2017;39:194-9.

26. Fan SJ, Ma Y, Chang EY, Bydder GM, Du J. Inversion recovery ultrashort echo time imaging of ultrahort $\mathrm{T} 2$ tissue components in ovine brain at 3T: a sequential D2O exchange study. NMR Biomed 2017;30:e3767.

27. Fan SJ, Ma Y, Searleman A, Zhu Y, Bydder GM, Du J. Yet more evidence that myelin protons can be directly imaged with ultrashort echo time (UTE) sequences on a clinical 3T scanner: bi-component $\mathrm{T} 2$ * analysis of native and deuterated ovine brain specimens. Magn Reson Med 2018;80:538-47.

28. Jang H, Ma Y, Searleman AC, Carl M, Corey-Bloom J, Chang EY, Du J. Inversion recovery UTE based volumetric myelin imaging in human brain using interleaved hybrid encoding. Magn Reson Med 2020;83:950-61.

29. Larson PE, Conolly SM, Pauly JM, Nishimura DG. Using adiabatic inversion pulses for long-T2 suppression in ultrashort echo time (UTE) imaging. Magn Reson Med 2007;58:952-61.

30. Carl M, Bydder GM, Du J. UTE imaging with simultaneous water and fat signal suppression using a timeefficient multi-spoke inversion recovery pulse sequence. Magn Reson Med 2016;76:577-82.

31. Ma YJ, Searleman A, Jang H, Fan SJ, Xue Y, Cai Z, Chang EY, Corey-Bloom J, Du J. Volumetric Imaging of Myelin 
in Vivo using 3D Inversion-Recovery Ultrashort Echo Time Cones (3D IR-UTE-Cones) Magnetic Resonance Imaging. NMR Biomed 2020. In press.

32. Seifert AC, Li C, Wilhelm MJ, Wehrli SL, Wehrli FW. Towards quantification of myelin by solid-state MRI of the lipid matrix protons. Neuroimage 2017;163:358-67.

33. Larocca JN, Norton WT. Isolation of myelin. Curr Protoc Cell Biol 2007: Chapter 3, Unit 3.25.

34. Ma YJ, Searleman A, Jang H, Wong J, Chang EY, Corey-Bloom J, Bydder GM, Du J. Whole-brain myelin mapping using 3D double echo sliding inversion recovery ultrashort echo time (DESIRE UTE) MRI. Radiology 2020;294:362-74.

35. Larson PE, Gurney PT, Nayak K, Gold GE, Pauly JM, Nishimura DG. Designing long-T2 suppression pulses for ultrashort echo time imaging. Magn Reson Med 2006;56:94-103.

36. Weiger M, Froidevaux R, Brunner DO, Rosler M, Pruessmann KP. Advances in Direct Myelin Imaging. In: Proc ISMRM 27th Annual Meeting. Montreal, Canada; 2019 p. 1118.

37. Jang H, Wei Z, Wu M, Ma Y, Chang EY, Corey-Bloom J, Du J. Improved volumetric myelin imaging in human brain using $3 \mathrm{D}$ dual echo inversion recovery-prepared UTE with complex echo subtraction. Magn Reson Med 2020;83:1168-77.

38. Carl M, Chiang J-TA. Investigations of the origin of phase differences seen with ultrashort TE imaging of short T2 meniscal tissue. Magn Reson Med 2012;67:991-1003.

39. Port JD. Why we DESIRE to directly image brain myelin using MRI. Radiology 2020;294:375-6.

40. Lustig M, Donoho D, Pauly JM. Sparse MRI: The application of compressed sensing for rapid MR imaging. Magn Reson Med 2007;58:1182-95.

41. Guglielmetti C, Boucneau T, Cao P, Van der Linden A, Larson PEZ, Chaumeli MM. Longitudinal evaluation of demyelinated lesions in a multiple sclerosis model using ultrashort echo time magnetization transfer (UTE-MT) imaging. Neuroimage 2020;208:116415.

42. Ramani A, Aliev AE, Barker GJ, Tofts PS. Another approach to protons with constricted mobility in white matter: pilot studies using wideline and highresolution NMR spectroscopy. Magn Reson Imaging 2003;21:1039-43.

43. Harkins KD, Does MD. Myelin UTE imaging, to be or not to be? In: Proc. of the ISMRM 27th Annual Meeting \& Exhibition. Montreal, Canada; 2019. p. 4895.

44. Norton WT, Cammer W. Isolation and Characterization of Myelin. In: Morell P. editor. Myelin. Plenum, New York, 1984;155.

45. Ma Y, Jang H, Wei Z, Cai Z, Xue Y, Chang EY, CoreyBloom J, Du J. Quantitative Myelin Mapping in Human Brain Using a Short TR Adiabatic Inversion Recovery Prepared Ultrashort Echo Time (STAIR-UTE) Sequence. Proc 28th Ann Mtg ISMRM, Sydney, Australia, 2020, (accepted for oral presentation).

46. Ma J, Jang H, Wei Z, Cai Z, Xue Y, Chang EY, CoreyBloom J, Du J. Robust Morphological Myelin Imaging Using a Short TR Adiabatic Inversion Recovery Prepared Ultrashort Echo Time (STAIR-UTE) Sequence. Proc 28th Ann Mtg ISMRM Sydney, Australia, 2020, (accepted for oral presentation).

47. Suzuki S, Sakai O, Jara H. Combined volumetric T1, T2 and secular-T2 quantitative MRI of the brain: age-related global changes (preliminary results). Magn Reson Imaging 2006;24:877-87.

48. Cheng HL, Stikov N, Ghugre NR, Wright GA. Practical medical applications of quantitative MR relaxometry. J Magn Reson Imaging 2012;36:805-24.

49. Rooney WD, Johnson G, Li X, Cohen ER, Kim SG, Ugurbil K, Spinger CS. Magnetic field and tissue dependencies of human brain longitudinal $1 \mathrm{H} 2 \mathrm{O}$ relaxation in vivo. Magn Reson Med 2007;57:308-18.

50. Stikov N, Boudreau M, Levesque IR, Tardif CL, Barral JK, Pike GB. On the accuracy of T1 mapping: searching for common ground. Magn Reson Med 2015;73:514-22.

51. MacKay A, Laule C, Vavasour I, Bjarnason T, Kolind S, Madler B. Insights into brain microstructure from the T2 distribution. Magn Reson Imaging 2006;24:515-25.

52. Hwang D, Kim DH, Du YP. In vivo multi-slice mapping of myelin water content using $\mathrm{T}^{*}$ * decay. NeuroImage 2010;52:198-204.

53. Oh SH, Bilello M, Schindler M, Markowitz CE, Detre JA, Lee J. Direct visualization of short transverse relaxation time component (ViSTa). NeuroImage 2013;83:485-92.

54. Münzel EJ, Williams A. Promoting remyelination in multiple sclerosis - recent advances. Drugs 2013;73:2017-29.

55. Mi S, Hu B, Hahm K, Luo Y, Kam Hui ES, Yan Q, Wong WM, Wang L, Su H, Chu TH, Guo J, Zhang W, So KF, Pepinsky B, Shao Z, Graff C, Garber E, Jeng V, Wu EX, Wu W. LINGO-1 antagonist promotes spiral cord remyelination and axonal integrity in MOG-induced experimental autoimmune encephalomyelitis. Nat Med 2007;13:1228-33.

56. Pirko I, Ciric C, Gameez J, Bierber AJ, Warrington AE, 
Johnson AJ, Hansen DP, Pease LR, Macura SI Rodriguez M. A human antibody that promotes remyelination enters the CNS and decreases lesion load as detected by T2weighted spinal cord MRI in a virus-induced murine model of MS. FASEB J 2004;18:1577-9.

57. Watzlawik JO, Warrington AE, Roderiguez M. PDGF is

Cite this article as: $\mathrm{Ma} \mathrm{YJ}$, Jang $\mathrm{H}$, Chang EY, Hiniker A, Head BP, Lee RR, Corey-Bloom J, Bydder GM, Du J. Ultrashort echo time (UTE) magnetic resonance imaging of myelin: technical developments and challenges. Quant Imaging Med Surg 2020;10(6):1186-1203. doi: 10.21037/qims-20-541 required for remyelination-promoting $\operatorname{IgM}$ stimulation of oligodentrocyte progenitor cell proliferation. PLoS One 2013;8:e55149.

58. Chang A, Tourtellotte WW, Rudick R, Trapp BD. Premyelinating oligodentrocytes in chronic lesions of multiple sclerosis. N Engl J Med 2002;346:165-73. 